\section{Determinantes individuais e contextuais associados à mortalidade infantil nas capitais brasileiras: uma abordagem multinível}

\author{
Individual and contextual determinants of infant \\ mortality in Brazilian state capitals: a multilevel \\ approach
}

\section{Determinantes individuales y contextuales asociados a la mortalidad infantil en las capitales brasileñas: un abordaje multinivel}

\section{Resumo}

A pesquisa buscou identificar os fatores de risco individuais e contextuais da assistência à saúde, suas interações e diferenciais regionais na determinação da mortalidade infantil nas capitais brasileiras. Trata-se de um estudo caso-controle, no qual considerou-se casos os 7.470 óbitos infantis ocorridos em 2012 nas 27 capitais do país, registrados no Sistema de Informações sobre Mortalidade (SIM) e pareados com o Sistema de Informações de Nascidos Vivos (SINASC) por meio do linkage, e 24.285 controles obtidos mediante amostra dos nascidos sobreviventes entre 2011 e 2012 do universo de 1.424 .691 nascimentos. As variáveis explicativas do nível individual corresponderam às informações disponibilizadas pelo SINASC, e a variável contextual consistiu um indice de qualidade da assistência hospitalar relativo aos 702 serviços de saúde onde ocorreram os nascimentos. Empregou-se o modelo logístico multinível e a análise de interação. Os principais determinantes da mortalidade infantil foram os fatores biológicos (baixo peso ao nascer, prematuridade, malformação congênita, asfixia grave/moderada e raça/cor), mediados pelos socioeconômicos maternos (escolaridade, estado civil e ocupação), e pela insuficiência de pré-natal. Realizar baixo número de consultas pré-natais representou risco para a mortalidade infantil independentemente da qualidade do serviço, à exceção das capitais da Região Sul. Na interação entre renda e pré-natal, observou-se que realizar poucas consultas e nascer em cidades com alta renda representaram risco maior quando comparados aos nascimentos em capitais de baixa renda $(O R=0,68)$. A análise multinível evidenciou desigualdades regionais nos modelos de risco e reiterou a importância dos determinantes biológicos com mediação dos fatores socioeconômicos e assistenciais na mortalidade infantil.

Mortalidade Infantil; Fatores de Risco; Determinantes Sociais da Saúde; Análise Multinível
Lívia Teixeira de Souza Maia 1

Wayner Vieira de Souza 2

Antonio da Cruz Gouveia Mendes 2

doi: 10.1590/0102-311X00057519

\section{Correspondência}

L. T. S. Maia

Universidade Federal de Pernambuco.

Rua Alto do Reservatório $s / n$, Vitória de Santo Antão, PE 55608-680, Brasil.

livia_tsouza@yahoo.com.br

1 Universidade Federal de Pernambuco, Vitória de Santo Antão, Brasil.

2 Instituto Aggeu Magalhães, Fundação Oswaldo Cruz, Recife, Brasil. 


\section{Introdução}

A mortalidade infantil representa um importante indicador de saúde pública por tratar-se de mortes precoces e, em sua maioria, evitáveis. O óbito infantil ocorre como consequência de uma combinação de fatores biológicos, sociais, culturais e de falhas do sistema de saúde e, portanto, as intervenções dirigidas à sua redução dependem tanto de mudanças estruturais relacionadas às condições de vida da população, assim como de ações diretas definidas pelas políticas públicas de saúde 1 .

A maioria dos estudos sobre o tema evidencia que, apesar das causas perinatais serem importantes determinantes da mortalidade infantil, as precárias condições socioeconômicas ainda interferem significativamente na ocorrência destes óbitos, revelando problemas sociais e dificuldades de acesso aos serviços 2,3. Ademais, a despeito dos avanços logrados nas últimas décadas, ainda persistem obstáculos tanto no acesso como na qualidade da atenção materno-infantil tais como: a falta de conexão entre os serviços de pré-natal e de assistência ao parto, problemas na organização dos serviços, carência e má distribuição de vagas e leitos obstétricos 4,5 .

A rede de determinantes da mortalidade infantil, portanto, é complexa e resulta de uma interação entre fatores de várias dimensões 6,7. Nesse sentido, vem aumentando o interesse sobre o efeito das características dos locais de residência das crianças e suas famílias, além das características individuais que poderiam afetar a mortalidade infantil e seus componentes 8,9 .

A literatura atual tem apresentado estratégias analíticas visando a uma aproximação mais concreta dos fatores implicados na determinação desse evento 9,10,11. A crescente multiplicidade da estrutura dos dados de estudos epidemiológicos e a disponibilidade de programas estatísticos têm aumentado a utilização dos modelos multiníveis 11 . Esses surgem como uma alternativa aos modelos multivariados tradicionais por considerar a natureza hierárquica intrínseca aos dados e analisar a autocorrelação entre os fatores de risco nos níveis de agregação 12,13,14.

Embora essa metodologia já venha sendo utilizada internacionalmente e no país para o estudo da mortalidade infantil 10,11,15,16, até o momento nenhuma pesquisa publicada explorou os determinantes desta mortalidade nas capitais brasileiras sob o enfoque de análise multinível incluindo o nível da assistência à saúde.

Nessa perspectiva, este estudo teve como objetivo identificar os fatores de risco individuais e contextuais da assistência à saúde, suas interações e diferenciais regionais na determinação da mortalidade infantil nas capitais brasileiras.

\section{Metodologia}

Realizou-se um estudo de caso-controle com abordagem multinível. Foram analisados os óbitos de menores de um ano ocorridos entre 1o de janeiro e 31 de dezembro de 2012 registrados no Sistema de Informações sobre Mortalidade (SIM), e os nascidos vivos entre 1o de janeiro de 2011 e 31 de dezembro de 2012 contidos no Sistema de Informações de Nascidos Vivos (SINASC), de residentes nas capitais brasileiras.

Para a definição dos casos e dos controles foi realizado linkage (determinístico e probabilístico) entre o SIM e o SINASC ${ }^{17}$. O linkage determinístico usou uma variável unificadora comum aos dois sistemas (o número da Declaração de Nascido Vivo - DNV). Para os registros não pareados nessa fase recorreu-se ao relacionamento probabilístico por meio de rotinas automatizadas (padronização, relacionamento e combinação dos arquivos) para sua execução, baseando-se em campos comuns presentes em ambos os bancos de dados com o objetivo de identificar a probabilidade de um par de registros pertencer a um mesmo indivíduo. Todo o processamento da etapa probabilística foi executado com o auxílio do software Reclink III versão 3.0.4.4005 (http://reclink.sourceforge.net/).

Os casos corresponderam aos óbitos infantis pareados à respectiva DNV. Os controles foram obtidos mediante amostra dos nascidos vivos que não foram a óbito, logo não pareados com o SIM. O tamanho da amostra foi calculado sendo necessária a proporção de três controles para cada caso.

Para garantir que os controles fossem selecionados da mesma população dos casos, adotou-se como critério a capital de residência. Foram excluídas as crianças com peso ao nascer inferior a $500 \mathrm{~g}$ visando a evitar possível superestimação dos valores da odds ratio (OR) 18 . 
Após o linkage sucedeu-se a verificação do estabelecimento de nascimento da criança com base no código do Cadastro Nacional de Estabelecimentos de Saúde (CNES) registrado no SINASC (CODESTAB). Valendo-se da identificação dos serviços, consultou-se a base de dados do CNES para tabulação e análise das informações referentes às variáveis do nível da assistência. Os registros de óbitos e nascimentos para os quais a unidade de saúde não foi localizada foram excluídos do estudo.

Considerou-se como variável dependente o óbito de menor de um ano, e as independentes foram alocadas em três níveis: individual, relativos à assistência à saúde e às capitais.

As variáveis do nível individual consistiram nos dados disponibilizados pelo SINASC para cada nascido vivo. Foram utilizadas as informações maternas (idade, escolaridade, ocupação, estado civil, raça/cor e paridade - número de filhos vivos e mortos em gestações anteriores), relativas à criança (sexo, presença de malformação congênita, peso ao nascer, idade gestacional e índice de apgar no primeiro minuto) e relacionadas à gravidez e ao parto (número de consultas de pré-natal, tipo da gravidez e tipo de parto).

A qualidade do estabelecimento de nascimento da criança foi definida como variável contextual da atenção à saúde. Para tanto, tomou-se por base o Índice de Completude da Qualidade da Assistência Hospitalar Materna e Neonatal proposto por Silva et al. 19. Nessa pesquisa, porém, utilizaram-se apenas os dados do CNES para o mês de dezembro de 2012, uma vez que foram incluídos os serviços da rede privada.

Assim, foram analisadas 19 variáveis agregadas em 4 subdimensões, a saber: I - Porte: número de leitos obstétricos e neonatais; II - Complexidade: número de leitos UTI (adulto, pediátrica e neonatal), incubadora, habilitação alto risco, nutrição enteral, atendimento fissura labiopalatal, tratamento do hipotireoidismo congênito, fenilcetonúria, hemoglobinopatias e fibrose cística; III - Práticas obstétricas e neonatais: alojamento conjunto, banco de leite, Hospital Amigo da Criança, atendimento à aids, cuidados intermediários neonatais, laqueadura e busca ativa de órgãos; e IV - Práticas epidemiológicas: comissão de investigação de óbitos e comissão de notificação e investigação de doenças.

Uma vez atribuída a pontuação a cada indicador, realizou-se o somatório dos valores dos indicadores simples correspondentes a cada subdimensão e o somatório destas compôs o Indicador Sintético de Completude (ISC). Para a definição das categorias de completude, empregou-se o método de K-means 20 utilizado para a criação de agrupamentos automáticos de dados segundo seu grau de semelhança (clusters), agrupando as unidades de nascimento das crianças em três clusters - Baixa (0,04,4), Intermediária $(4,6-8,0)$ e Alta $(8,2-14,6)$.

Para o contexto das capitais adotou-se o componente de renda per capita do Índice de Desenvolvimento Humano Municipal (IDH-M) do ano de 2010 21, agregado também pelo método K-means, em três categorias: renda alta $(0,850-0,876)$, renda intermediária $(0,789-0,841)$ e renda baixa $(0,723-$ $0,784)$. Uma vez que a capital de residência da criança foi tomada como critério de seleção na amostra de controles, a relação entre a renda e o óbito infantil foi explorada apenas na análise de interação.

$\mathrm{Na}$ análise dos modelos multivariados optou-se pela agregação das capitais em suas respectivas macrorregiões, resultando em seis modelos, incluindo um modelo para o conjunto das 27 cidades.

A análise univariada verificou a associação entre o desfecho e cada variável independente, sendo calculada a OR bruta e seus respectivos intervalos de 95\% de confiança (IC95\%) e significância estatística (valor de p) com base no teste de qui-quadrado $\left(\chi^{2}\right)$. As variáveis independentes que apresentaram significância estatística abaixo de $20 \%$ ( $<<0,20$ ) foram incluídas para a análise multivariada. Ademais, a qualidade da informação também foi considerada, tendo sido excluídas as variáveis que apresentaram proporção de campos não preenchidos/ignorados superior a $20 \%$ já nessa etapa.

O modelo multinível foi ajustado usando-se modelos lineares generalizados com função de ligação logito (regressão logística), considerando as informações referentes aos indivíduos como primeiro nível, a unidade de nascimento da criança como segundo nível e ajustados pela cidade (terceiro nível).

Também para ajuste do modelo optou-se pelo método stepwise, iniciando com todas as variáveis significativas na bivariada e, em seguida, retirando aquelas cuja significância estatística apresentou valores acima de 10\% ( $\mathrm{p}>0,10)$ (processo backward). Para avaliar a qualidade dos modelos de regressão, foram utilizados o critério de Akaike (AIC) e o critério de informação Bayesiano (BIC), em cada passo da análise multivariada. Sendo considerados os melhores modelos aqueles que resultaram em menores valores de AIC e BIC. 
Ainda como desdobramento do modelo multinível, buscou-se testar o efeito de interação entre variáveis explicativas do nível contextual sobre o individual. Em função da relevância dos aspectos referentes à assistência à saúde materno-infantil no desfecho estudado, investigaram-se as interações entre consultas de pré-natal e a completude dos serviços.

No modelo agregado das capitais testou-se a interação entre o número de consultas de pré-natal e a renda. Os valores das OR ajustadas pelo nível dois (estabelecimento) e nível três (cidade) foram analisados e comparados aos resultados obtidos antes da análise de interação. Para tanto, adicionouse o termo de interação ao modelo logito de interceptação aleatória de três níveis executado pelo GLLAMM por meio do Stata versão 12.0 (https://www.stata.com).

A pesquisa usou dados do SIM e SINASC fornecidos pelo Ministério da Saúde mediante parecer técnico, termo de cessão e utilização dos bancos de dados, além da assinatura do termo de responsabilidade pelos pesquisadores. Foi também aprovada pelo Comitê de Ética em Pesquisa (Registro no CAAE - 35632414.5.0000.5190).

\section{Resultados}

O trabalho partiu de um universo de 9.001 óbitos de menores de um ano e 1.424.691 nascidos vivos no total das capitais brasileiras. Concluídas as etapas de processamento dos dados, a população do estudo foi composta por 7.470 casos e 24.285 controles nascidos em 702 serviços de saúde (Figura 1).

A Figura 1 sistematiza as etapas de processamento para a definição dos grupos de casos e controles, explicitando as perdas e exclusões de registros em cada uma das etapas.

No tocante à caracterização dos casos e controles em relação às variáveis maternas, observou-se um predomínio de mães com idades entre 20 e 34 anos, com a proporção de mães adolescentes maior no grupo de casos. Entre os óbitos constatam-se maiores percentuais de mulheres solteiras, separadas e viúvas. Quanto à escolaridade, entre $70 \%$ e $80 \%$ das mães possuíam mais de oito anos de estudos. Entre os casos prevaleceram mães sem ocupação remunerada (Tabela 1).

A maioria das mães é de raça/cor não branca, exceto na Região Sul. Verifica-se maior número de primíparas dentre os grupos, com alto percentual de multíparas no Norte. A maior parte das mães não teve nenhum filho nascido morto, porém, ressalta-se a elevada proporção de registros ignorados desta variável no Norte (34,2\%), impossibilitando a sua inclusão na análise univariada (Tabela 1).

No que diz respeito às características das crianças, percebeu-se um predomínio de recém-nascidos do sexo masculino. Em relação ao peso ao nascer, mais de $90 \%$ dos sobreviventes apresentaram peso maior que $2.500 \mathrm{~g}$. No conjunto das capitais a prevalência de baixo peso entre os controles foi de $8,8 \%$, já entre os casos foi de $66,1 \%$ (Tabela 1 ).

De maneira análoga, a prevalência de prematuridade foi mais alta entre as crianças que foram a óbito. No conjunto das capitais a proporção de nascidos vivos que apresentaram asfixia grave a moderada no $1^{\circ}$ minuto (apgar 0 a 4 ) foi de $34,9 \%$ nos casos. Também constatou-se maior prevalência de malformações congênitas no grupo de casos (Tabela 1).

Em relação à gestação e ao parto, predominaram as gestações únicas e os partos cesáreos. Observaram-se diferenças importantes relativas ao pré-natal. No agregado das capitais constatou-se um maior número de mães dos sobreviventes que realizaram mais de sete consultas (61,1\%), já nos casos foram 31,3\% (Tabela 1).

Os nascimentos ocorreram predominantemente em unidades de completude intermediária, exceto no Sul onde foram verificados mais altos porcentuais de partos em unidades de alta completude. Dentre os casos, destaca-se a superioridade de nascimentos em serviços de alta completude $(58,1 \%)$ (Tabela 1).

A Tabela 2 apresenta os valores da OR bruta e dos respectivos valores da significância estatística resultantes da análise bivariada entre o óbito infantil e as variáveis independentes do estudo, para o conjunto das capitais brasileiras e seus agregados nas respectivas macrorregiões.

$\mathrm{Na}$ análise univariada entre o óbito infantil e as variáveis independentes não foram observadas associações significativas apenas para: estado civil nas regiões Sul e Centro-oeste; escolaridade, ocupação e número de filhos mortos no Centro-oeste; e tipo de parto nas capitais do Sul. As demais variáveis, portanto, foram incluídas no modelo multinível (Tabela 2). 


\section{Figura 1}

Consolidado das etapas de processamento (linkage e identificação dos estabelecimentos de nascimento das crianças) para a definição dos grupos de casos e controles no conjunto das capitais brasileiras, 2012.

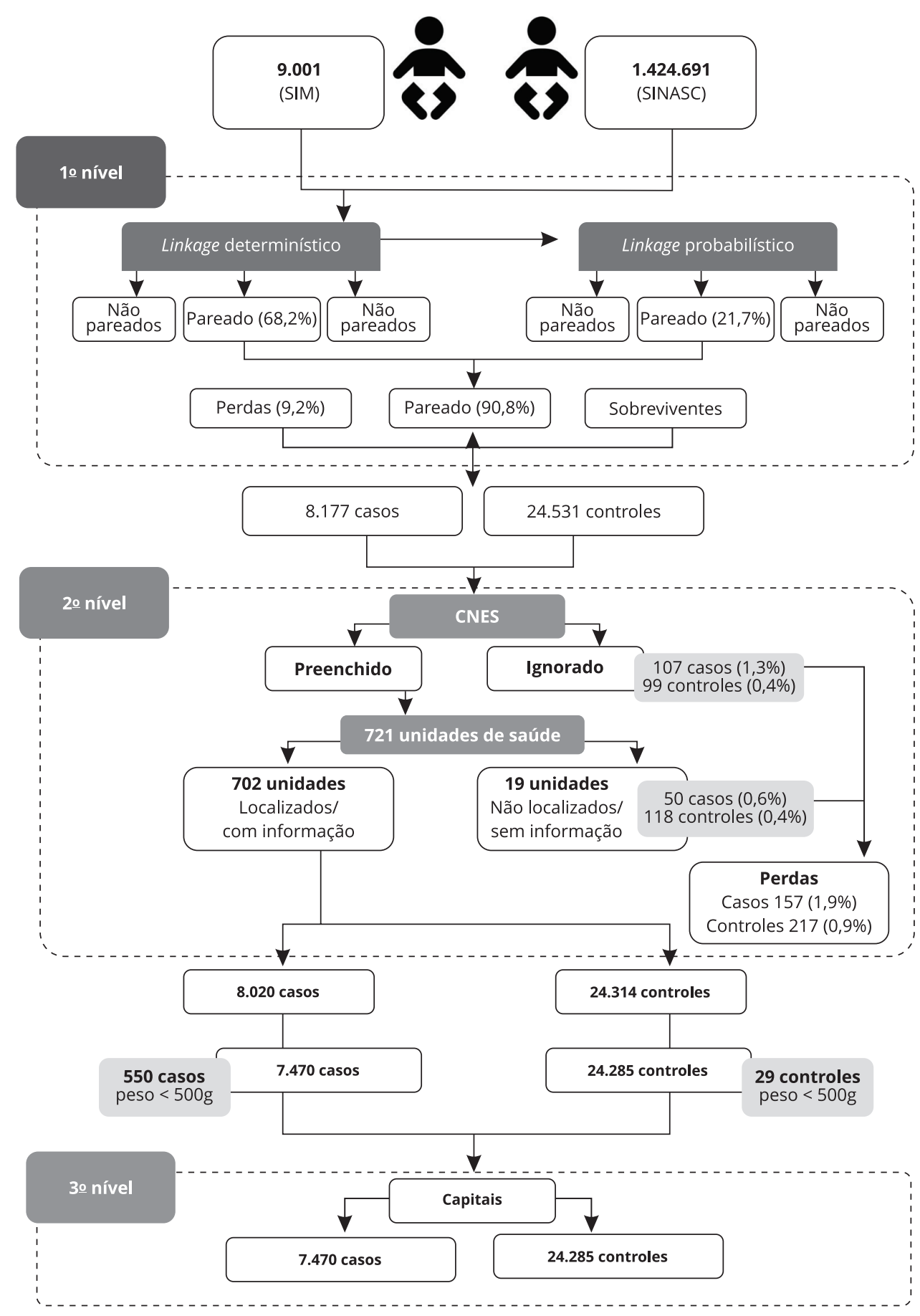

CNES: Cadastro Nacional de Estabelecimentos de Saúde; SIM: Sistema de Informações sobre Mortalidade; SINASC: Sistema de Informações de Nascidos Vivos. 
Tabela 1

Proporção de casos (C1) e controles (C2) segundo as variáveis independentes individuais e contextuais para as capitais brasileiras agregadas nas respectivas macrorregiões, 2012.

\begin{tabular}{|c|c|c|c|c|c|c|c|c|c|c|c|c|}
\hline \multirow[t]{2}{*}{ Variáveis } & \multicolumn{2}{|c|}{ Norte } & \multicolumn{2}{|c|}{ Nordeste } & \multicolumn{2}{|c|}{ Sudeste } & \multicolumn{2}{|c|}{ Sul } & \multicolumn{2}{|c|}{ Centro-oeste } & \multicolumn{2}{|c|}{27 capitais } \\
\hline & C1 & C2 & C1 & C2 & C1 & C2 & C1 & C2 & C1 & C2 & C1 & C2 \\
\hline \multicolumn{13}{|l|}{ Maternas } \\
\hline \multicolumn{13}{|l|}{ Idade da mãe (anos) } \\
\hline$<20$ & 26,9 & 21,5 & 21,5 & 17,6 & 19,8 & 14,0 & 18,4 & 14,4 & 20,5 & 14,5 & 21,4 & 16,3 \\
\hline $20-34$ & 63,1 & 69,9 & 64,1 & 71,0 & 63,2 & 70,4 & 64,2 & 68,6 & 64,7 & 72,5 & 63,6 & 70,6 \\
\hline 35 e mais & 10,0 & 8,6 & 14,4 & 11,3 & 17,0 & 15,6 & 17,4 & 17,0 & 14,9 & 13,1 & 15,0 & 13,1 \\
\hline \multicolumn{13}{|l|}{ Estado civil } \\
\hline Casada, união estável & 46,6 & 49,5 & 55,8 & 55,4 & 39,3 & 43,8 & 45,2 & 45,2 & 56,8 & 59,3 & 47,2 & 49,7 \\
\hline Solteira, separada, viúva & 52,9 & 49,3 & 42,2 & 43,4 & 60,3 & 55,7 & 54,1 & 54,2 & 41,4 & 39,3 & 51,8 & 49,4 \\
\hline \multicolumn{13}{|l|}{ Escolaridade (anos) } \\
\hline$<8$ & 29,9 & 26,2 & 27,7 & 26,2 & 23,1 & 18,5 & 21,6 & 18,2 & 19,6 & 17,9 & 25,0 & 21,8 \\
\hline 8 e mais & 69,7 & 72,7 & 71,3 & 72,6 & 76,3 & 81,1 & 76,5 & 81,6 & 79,9 & 79,9 & 74,3 & 77,4 \\
\hline \multicolumn{13}{|l|}{ Ocupação } \\
\hline Com ocupação & 26,2 & 45,3 & 32,3 & 60,6 & 41,4 & 67,8 & 52,5 & 60,5 & 44,9 & 40,7 & 37,5 & 56,8 \\
\hline Sem ocupação & 70,5 & 49,6 & 61,9 & 29,2 & 55,2 & 23,0 & 47,3 & 30,1 & 50,1 & 49,9 & 58,4 & 34,8 \\
\hline \multicolumn{13}{|l|}{ Raça/Cor } \\
\hline Branca & 12,0 & 16,0 & 16,4 & 22,2 & 41,9 & 51,5 & 79,5 & 85,1 & 35,2 & 41,7 & 31,6 & 38,7 \\
\hline Não branca & 86,9 & 84,0 & 79,9 & 77,8 & 57,8 & 48,5 & 20,5 & 14,9 & 63,0 & 58,3 & 66,9 & 61,3 \\
\hline \multicolumn{13}{|l|}{ Número de filhos vivos } \\
\hline 0 & 44,3 & 24,3 & 49,8 & 37,6 & 48,5 & 47,6 & 56,0 & 50,1 & 48,9 & 36,8 & 48,6 & 40,2 \\
\hline 1 & 26,6 & 28,9 & 26,6 & 29,6 & 25,1 & 29,7 & 20,7 & 17,7 & 26,7 & 29,5 & 25,7 & 29,7 \\
\hline 2 e mais & 29,0 & 27,6 & 23,1 & 20,2 & 25,9 & 20,4 & 23,3 & 18,7 & 22,9 & 24,0 & 25,2 & 21,8 \\
\hline \multicolumn{13}{|l|}{ Número de filhos mortos } \\
\hline 0 & 76,9 & 49,9 * & 73,8 & 63,0 & 75,8 & 81,4 & 80,5 & 87,4 & 78,2 & 69,0 & 76,0 & 70,2 \\
\hline 1 & 18,2 & 13,2 * & 17,9 & 13,8 & 17,5 & 11,9 & 14,6 & 9,9 & 14,5 & 13,2 & 17,2 & 12,6 \\
\hline 2 e mais & 4,7 & 3,6 * & 7,2 & 0,3 & 5,9 & 3,0 & 4,7 & 2,3 & 5,1 & 3,2 & 5,9 & 3,4 \\
\hline \multicolumn{13}{|l|}{ Criança } \\
\hline \multicolumn{13}{|l|}{ Sexo } \\
\hline Feminino & 46,0 & 49,9 & 45,3 & 48,4 & 44,5 & 48,1 & 43,5 & 48,0 & 43,0 & 49,5 & 44,7 & 48,6 \\
\hline Masculino & 53,8 & 50,1 & 54,2 & 51,6 & 55,3 & 51,9 & 55,8 & 52,0 & 57,0 & 50,5 & 55,0 & 51,4 \\
\hline \multicolumn{13}{|l|}{ Peso ao nascer } \\
\hline Muito baixo peso & 42,5 & 1,1 & 50,2 & 1,1 & 46,3 & 1,3 & 48,9 & 1,2 & 45,3 & 1,1 & 46,7 & 1,2 \\
\hline Baixo peso & 21,8 & 6,9 & 17,7 & 7,9 & 18,9 & 8,0 & 19,1 & 7,2 & 21,3 & 6,7 & 19,3 & 7,6 \\
\hline Normal & 35,7 & 92,0 & 32,2 & 91,0 & 34,8 & 90,7 & 32,0 & 91,5 & 33,4 & 92,2 & 33,9 & 91,2 \\
\hline \multicolumn{13}{|l|}{ Idade gestacional } \\
\hline Pré-termo & 62,6 & 11,7 & 63,9 & 11,4 & 63,0 & 11,0 & 65,4 & 10,3 & 64,1 & 10,6 & 63,4 & 11,1 \\
\hline A termo & 34,9 & 81,3 & 32,6 & 81,7 & 36,5 & 88,5 & 33,9 & 88,7 & 35,2 & 85,4 & 34,9 & 85,5 \\
\hline \multicolumn{13}{|l|}{ Apgar no 1ㅇ minuto } \\
\hline $0-4$ & 28,4 & 1,3 & 37,8 & 2,5 & 35,5 & 2,4 & 38,8 & 3,4 & 33,2 & 1,7 & 34,9 & 2,2 \\
\hline $5-7$ & 23,3 & 7,6 & 25,2 & 10,0 & 26,4 & 9,5 & 24,2 & 9,2 & 29,2 & 10,3 & 25,8 & 9,4 \\
\hline $8-10$ & 40,3 & 89,8 & 30,4 & 86,5 & 33,8 & 87,8 & 33,9 & 87,3 & 34,3 & 87,1 & 34,0 & 87,7 \\
\hline Malformação congênita & & & & & & & & & & & & \\
\hline Sim & 11,2 & 0,4 & 12,0 & 0,6 & 26,4 & 1,1 & 22,4 & 1,0 & 13,3 & 0,5 & 18,4 & 0,8 \\
\hline Não & 82,4 & 95,1 & 79,6 & 94,0 & 72,3 & 97,7 & 74,4 & 98,4 & 79,0 & 94,8 & 76,8 & 96,0 \\
\hline
\end{tabular}

(continua) 
Tabela 1 (continuação)

\begin{tabular}{|c|c|c|c|c|c|c|c|c|c|c|c|c|}
\hline \multirow[t]{2}{*}{ Variáveis } & \multicolumn{2}{|c|}{ Norte } & \multicolumn{2}{|c|}{ Nordeste } & \multicolumn{2}{|c|}{ Sudeste } & \multicolumn{2}{|c|}{ Sul } & \multicolumn{2}{|c|}{ Centro-oeste } & \multicolumn{2}{|c|}{27 capitais } \\
\hline & C1 & $\mathrm{C} 2$ & $\mathrm{C} 1$ & $\mathrm{C2}$ & C1 & $\mathrm{C2}$ & C1 & C2 & C1 & $\mathrm{C} 2$ & $\mathrm{C} 1$ & $\mathrm{C} 2$ \\
\hline \multicolumn{13}{|l|}{ Gravidez e parto } \\
\hline \multicolumn{13}{|l|}{ Tipo de gravidez } \\
\hline Única & 93,0 & 97,8 & 91,7 & 97,3 & 91,4 & 97,6 & 87,5 & 97,4 & 89,7 & 96,6 & 91,3 & 97,5 \\
\hline Dupla/Múltipla & 7,0 & 0,1 & 8,2 & 0,0 & 8,5 & 2,3 & 12,5 & 2,6 & 10,3 & 2,8 & 8,6 & 2,2 \\
\hline \multicolumn{13}{|c|}{$\begin{array}{l}\text { Número de consultas de pré- } \\
\text { natal }\end{array}$} \\
\hline $0-3$ & 39,7 & 18,1 & 37,6 & 14,2 & 25,6 & 6,5 & 23,8 & 5,6 & 24,7 & 8,8 & 30,9 & 10,6 \\
\hline 4 & 32,3 & 34,7 & 35,7 & 37,9 & 34,4 & 19,2 & 29,4 & 15,3 & 36,3 & 20,5 & 34,3 & 26,7 \\
\hline 7 e mais & 22,1 & 43,8 & 23,4 & 46,2 & 37,0 & 73,3 & 43,3 & 78,5 & 37,4 & 69,8 & 31,3 & 61,1 \\
\hline \multicolumn{13}{|l|}{ Tipo de parto } \\
\hline Cesáreo & 50,0 & 54,6 & 49,1 & 56,2 & 51,9 & 55,1 & 58,1 & 57,7 & 58,0 & 62,1 & 51,9 & 56,2 \\
\hline Vaginal & 50,0 & 45,3 & 50,9 & 43,5 & 48,0 & 44,8 & 41,9 & 42,2 & 42,0 & 37,5 & 48,1 & 43,6 \\
\hline \multicolumn{13}{|l|}{ Assistência } \\
\hline \multicolumn{13}{|c|}{ Completude do serviço } \\
\hline Alta & 21,7 & 11 & 46,7 & 26,1 & 40,3 & 35,2 & 58,1 & 40,7 & 41,2 & 24,2 & 40,1 & 28,0 \\
\hline Intermediária & 54,7 & 49,5 & 31,7 & 34,9 & 46,5 & 50,3 & 27,8 & 43 & 41,1 & 47,7 & 42,2 & 45,2 \\
\hline Baixa & 23,6 & 39,5 & 21,6 & 39 & 13,1 & 14,5 & 14,1 & 16,3 & 17,7 & 28,1 & 17,7 & 26,8 \\
\hline Total & 100,0 & 100,0 & 100,0 & 100,0 & 100,0 & 100,0 & 100,0 & 100,0 & 100,0 & 100,0 & 100,0 & 100,0 \\
\hline
\end{tabular}

Fonte: elaborada pelos autores com base em dados do Sistema de Informações sobre Mortalidade e Sistema de Informações de Nascidos Vivos, Secretaria de Vigilância em Saúde, Ministério da Saúde.

Nota: os percentuais calculados para cada variável têm como denominador o número total de óbitos e de nascidos vivos em cada região, incluindo os dados ignorados/não preenchidos; Apresentaram percentuais de incompletude entre 10 e $20 \%$ no grupo de controles as variáveis: ocupação materna (10,1\% - Norte); Número de filhos vivos (19,3\% - Norte; 12,2\% - Nordeste; 13,3\% - Sul); Número de filhos mortos (34,2\% - Norte; 18,9\% - Nordeste; $14,4 \%$ - Centro-oeste; $13,7 \%$ - 27 capitais); No grupo de casos nenhuma variável apresentou percentual de incompletude entre 10 e $20 \%$.

* Percentual de incompletude superior a $20 \%$.

O modelo final dos determinantes da mortalidade infantil do conjunto das capitais foi integrado pelo maior número de variáveis explicativas (13), seguido do modelo da Região Sudeste. Já a Região Sul é a que apresenta o menor número de fatores de risco na composição do seu modelo final (7). A variável tipo de parto foi a única que não permaneceu em nenhum modelo (Tabela 3 ).

Em relação às características maternas, observou-se que a idade da mãe esteve associada ao óbito infantil em todas as regiões, exceto no Sul. Os nascidos de mães adolescentes foram os que apresentaram maiores chances de apresentar o desfecho, principalmente no Centro-oeste $(\mathrm{OR}=3,58)$. Ser filho de mulher solteira, separada ou viúva representou fator de risco para o óbito infantil nas capitais brasileiras e nas regiões Norte e Sudeste (Tabela 3).

A baixa escolaridade materna esteve associada com o desfecho nas regiões Norte e Sul e no agregado das capitais. Na Região Sul, a chance de vir a óbito no 1o ano de vida entre os filhos de mães com menos de 8 anos de estudos foi $85 \%$ maior do que a dos filhos de mães com mais de 8 anos de estudos (Tabela 3).

A ausência de ocupação remunerada da mãe só não compôs o modelo de risco das regiões Sul e Centro-oeste. Nas demais, a chance de ser filho de mães sem ocupação remunerada foi maior entre os casos, com maior valor da OR observado na Região Nordeste $(2,83)$ (Tabela 3). Ser filho de mãe não branca consistiu em fator de risco para a mortalidade infantil em grande parte dos modelos finais, exceto nas regiões Norte e Sul (Tabela 3).

Nas regiões Norte e Sudeste e no conjunto das capitais ser filho de mães primíparas representou fator de proteção, já as multíparas consistiram em risco na maioria dos modelos. O número de filhos nascidos mortos permaneceu no modelo final apenas na Região Centro-oeste $(\mathrm{OR}=2,43)$ (Tabela 3 ). 


\section{Tabela 2}

Odds ratio bruta $\left(\mathrm{OR}_{\mathrm{b}}\right)$, respectivos intervalos de $95 \%$ de confiança (IC95\%) e significância estatística (valor de p) resultantes da análise univariada entre o óbito infantil e as variáveis independentes individuais e contextuais para as capitais brasileiras agregadas nas respectivas macrorregiões, 2012.

\begin{tabular}{|c|c|c|c|c|c|c|}
\hline Variáveis & $\begin{array}{c}\text { Norte } \\
\text { OR }_{b} \\
(I C 95 \%)\end{array}$ & $\begin{array}{c}\text { Nordeste } \\
\text { OR }_{b} \\
(I C 95 \%)\end{array}$ & $\begin{array}{l}\text { Sudeste } \\
\text { OR }_{\mathrm{b}} \\
(\mathrm{IC} 95 \%)\end{array}$ & $\begin{array}{c}\text { Sul } \\
\text { Or }_{b} \\
(I C 95 \%)\end{array}$ & $\begin{array}{c}\text { Centro-oeste } \\
\text { Or }_{\mathrm{b}} \\
(\mathrm{IC} 95 \%)\end{array}$ & $\begin{array}{c}27 \text { Capitais } \\
\text { OR }_{\mathrm{b}} \\
(\mathrm{IC} 95 \%)\end{array}$ \\
\hline \multicolumn{7}{|l|}{ Maternas } \\
\hline \multicolumn{7}{|l|}{ Idade da mãe (anos) } \\
\hline$<20$ & $\begin{array}{c}1,39 * \\
(1,19-1,62)\end{array}$ & $\begin{array}{c}1,35 * \\
(1,19-1,54)\end{array}$ & $\begin{array}{c}1,58 \text { * } \\
(1,41-1,76)\end{array}$ & $\begin{array}{c}1,36 \text { * } \\
(1,02-1,83)\end{array}$ & $\begin{array}{c}1,59 * \\
(1,29-1,95)\end{array}$ & $\begin{array}{c}1,46 \text { * } \\
(1,37-1,56)\end{array}$ \\
\hline $20-34$ & 1,00 & 1,00 & 1,00 & 1,00 & 1,00 & 1,00 \\
\hline 35 e mais & $\begin{array}{c}1,28 \text { * } \\
(1,04-1,60)\end{array}$ & $\begin{array}{c}1,41 \text { * } \\
(1,22-1,64)\end{array}$ & $\begin{array}{c}1,22 \text { * } \\
(1,09-1,36)\end{array}$ & $\begin{array}{c}1,10 * \\
(1,02-1,47)\end{array}$ & $\begin{array}{c}1,27 \text { * } \\
(1,06-1,95)\end{array}$ & $\begin{array}{c}1,27 \text { * } \\
(1,17-1,36)\end{array}$ \\
\hline \multicolumn{7}{|l|}{ Estado civil } \\
\hline Casada, união estável & 1,00 & 1,00 & 1,00 & 1,00 & 1,00 & 1,00 \\
\hline Solteira, separada, viúva & $\begin{array}{c}1,14 \text { * } \\
(1,05-1,30)\end{array}$ & $\begin{array}{c}0,97 \text { * } \\
(0,79-0,98)\end{array}$ & $\begin{array}{c}1,21 * \\
(1,11-1,31)\end{array}$ & $\begin{array}{c}0,99 * * \\
(0,80-1,24)\end{array}$ & $\begin{array}{c}1,11 * * \\
(0,94-1,29)\end{array}$ & $\begin{array}{c}1,11 * \\
(1,05-1,16)\end{array}$ \\
\hline \multicolumn{7}{|l|}{ Escolaridade (anos) } \\
\hline$<8$ & $\begin{array}{c}1,19 * \\
(1,03-1,38)\end{array}$ & $\begin{array}{c}1,07 \text { * } \\
(1,02-1,19)\end{array}$ & $\begin{array}{c}1,32 \text { * } \\
(1,20-1,46)\end{array}$ & $\begin{array}{c}1,37 * \\
(1,05-1,78)\end{array}$ & $\begin{array}{c}1,10 * * \\
(0,90-1,34)\end{array}$ & $\begin{array}{c}1,20 \text { * } \\
(1,13-1,27)\end{array}$ \\
\hline 8 e mais & 1,00 & 1,00 & 1,00 & 1,00 & 1,00 & 1,00 \\
\hline \multicolumn{7}{|l|}{ Ocupação } \\
\hline Com ocupação & 1,00 & 1,00 & 1,00 & 1,00 & 1,00 & 1,00 \\
\hline Sem ocupação & $\begin{array}{c}2,46 \text { * } \\
(1,13-3,22)\end{array}$ & $\begin{array}{c}3,97 \text { * } \\
(2,18-4,26)\end{array}$ & $\begin{array}{c}3,92 \text { * } \\
(3,73-4,17)\end{array}$ & $\begin{array}{c}1,43 \text { * } \\
(1,11-1,87)\end{array}$ & $\begin{array}{c}0,91 * * \\
(0,85-1,07)\end{array}$ & $\begin{array}{c}2,54 \text { * } \\
(2,24-2,79)\end{array}$ \\
\hline \multicolumn{7}{|l|}{ Raça/Cor } \\
\hline Branca & 1,00 & 1,00 & 1,00 & 1,00 & 1,00 & 1,00 \\
\hline Não Branca & $\begin{array}{c}1,39 \text { * } \\
(1,14-1,69)\end{array}$ & $\begin{array}{c}1,39 * \\
(1,22-1,59)\end{array}$ & $\begin{array}{c}1,47 * \\
(1,35-1,59)\end{array}$ & $\begin{array}{c}1,48 \text { * } \\
(1,12-1,95)\end{array}$ & $\begin{array}{c}1,28 * \\
(1,09-1,51)\end{array}$ & $\begin{array}{c}1,34 \text { * } \\
(1,16-1,41)\end{array}$ \\
\hline \multicolumn{7}{|l|}{ Número de filhos vivos } \\
\hline 0 & $\begin{array}{c}1,98 \text { * } \\
(1,68-2,33)\end{array}$ & $\begin{array}{c}1,49 * \\
(1,32-1,68)\end{array}$ & $\begin{array}{c}1,20 \text { * } \\
(1,09-1,33)\end{array}$ & $\begin{array}{c}1,68 \text { * } \\
(1,28-2,20)\end{array}$ & $\begin{array}{c}1,47 \text { * } \\
(1,22-1,77)\end{array}$ & $\begin{array}{c}1,40 \text { * } \\
(1,32-1,49)\end{array}$ \\
\hline 1 & 1,00 & 1,00 & 1,00 & 1,00 & 1,00 & 1,00 \\
\hline 2 e mais & $\begin{array}{c}1,14 \text { * } \\
(1,06-1,36)\end{array}$ & $\begin{array}{c}1,29 * \\
(1,11-1,48)\end{array}$ & $\begin{array}{c}1,50 * \\
(1,34-1,68)\end{array}$ & $\begin{array}{c}1,87 \text { * } \\
(1,35-2,59)\end{array}$ & $\begin{array}{c}1,05 \text { * } \\
(0,34-1,31)\end{array}$ & $\begin{array}{c}1,34 \text { * } \\
(1,24-1,44)\end{array}$ \\
\hline \multicolumn{7}{|l|}{ Número de filhos mortos } \\
\hline 0 & & 1,00 & 1,00 & 1,00 & 1,00 & 1,00 \\
\hline 1 & & $\begin{array}{c}1,11 \text { * } \\
(1,09-1,34)\end{array}$ & $\begin{array}{c}1,58 * \\
(1,31-1,82)\end{array}$ & $\begin{array}{c}1,60 * \\
(1,19-2,02)\end{array}$ & $\begin{array}{c}0,97 * * \\
(0,83-1,85)\end{array}$ & $\begin{array}{c}1,26 \text { * } \\
(1,08-1,54)\end{array}$ \\
\hline 2 e mais & & $\begin{array}{c}1,43 \text { * } \\
(1,37-1,76)\end{array}$ & $\begin{array}{c}2,09 * \\
(1,90-2,34)\end{array}$ & $\begin{array}{c}2,18 \text { * } \\
(1,57-2,86)\end{array}$ & $\begin{array}{c}1,42 * * \\
(0,97-1,61)\end{array}$ & $\begin{array}{c}1,58 \text { * } \\
(1,29-1,80)\end{array}$ \\
\hline
\end{tabular}

(continua) 
Tabela 2 (continuação)

\begin{tabular}{|c|c|c|c|c|c|c|}
\hline Variáveis & $\begin{array}{c}\text { Norte } \\
\text { OR }_{b} \\
(I C 95 \%)\end{array}$ & $\begin{array}{c}\text { Nordeste } \\
\text { OR }_{\mathrm{b}} \\
\text { (IC95\%) }\end{array}$ & $\begin{array}{l}\text { Sudeste } \\
\text { OR }_{\mathrm{b}} \\
(\mathrm{IC} 95 \%)\end{array}$ & $\begin{array}{c}\text { Sul } \\
\text { Or }_{b} \\
\text { (IC95\%) }\end{array}$ & $\begin{array}{c}\text { Centro-oeste } \\
\text { Or }_{\mathrm{b}} \\
\text { (IC95\%) }\end{array}$ & $\begin{array}{c}27 \text { Capitais } \\
\text { OR }_{\mathrm{b}} \\
\text { (IC95\%) }\end{array}$ \\
\hline \multicolumn{7}{|l|}{ Criança } \\
\hline \multicolumn{7}{|l|}{ Sexo } \\
\hline Feminino & 1,00 & 1,00 & 1,00 & 1,00 & 1,00 & 1,00 \\
\hline Masculino & $\begin{array}{c}1,17 \text { * } \\
(1,03-1,33)\end{array}$ & $\begin{array}{c}1,12 \text { * } \\
(1,02-1,24)\end{array}$ & $\begin{array}{c}1,15 \text { * } \\
(1,06-1,25)\end{array}$ & $\begin{array}{c}1,18 \text { * } \\
(0,95-1,47)\end{array}$ & $\begin{array}{c}1,31 \text { * } \\
(1,12-1,53)\end{array}$ & $\begin{array}{c}1,16 \text { * } \\
(1,11-1,23)\end{array}$ \\
\hline \multicolumn{7}{|l|}{ Peso ao nascer } \\
\hline Muito baixo peso & $\begin{array}{c}96,37 \text { * } \\
(69,5-133,6)\end{array}$ & $\begin{array}{c}128,40 * \\
(100,03-164,81)\end{array}$ & $\begin{array}{c}96,44 \text { * } \\
(79,27-117,30)\end{array}$ & $\begin{array}{c}112,46 \text { * } \\
(66,52-190,13)\end{array}$ & $\begin{array}{c}115,86 \text { * } \\
(77,87-172,40)\end{array}$ & $\begin{array}{c}107,10 \text { * } \\
(94,27-121,66)\end{array}$ \\
\hline Baixo peso & $\begin{array}{c}8,11 \text { * } \\
(6,65-9,89)\end{array}$ & $\begin{array}{c}6,32 \text { * } \\
(5,40-7,40)\end{array}$ & $\begin{array}{c}6,18 \text { * } \\
(5,45-7,00)\end{array}$ & $\begin{array}{c}7,52 \text { * } \\
(5,34-10,60)\end{array}$ & $\begin{array}{c}8,74 \text { * } \\
(6,87-11,13)\end{array}$ & $\begin{array}{c}6,82 * \\
(6,29-77,39)\end{array}$ \\
\hline Normal & 1,00 & 1,00 & 1,00 & 1,00 & 1,00 & 1,00 \\
\hline \multicolumn{7}{|l|}{ Idade gestacional } \\
\hline Pré-termo & $\begin{array}{c}12,42 \text { * } \\
(5,36-7,17)\end{array}$ & $\begin{array}{c}10,68 \text { * } \\
(8,12-13,44)\end{array}$ & $\begin{array}{c}13,85 \text { * } \\
(13,28-14,07)\end{array}$ & $\begin{array}{c}16,58 \text { * } \\
(13,38-19,15)\end{array}$ & $\begin{array}{c}10,44 \text { * } \\
(5,56-17,92)\end{array}$ & $\begin{array}{c}13,97 \text { * } \\
(12,83-14,39)\end{array}$ \\
\hline A termo & 1,00 & 1,00 & 1,00 & 1,00 & 1,00 & 1,00 \\
\hline \multicolumn{7}{|l|}{ Apgar no 10 minuto } \\
\hline $0-4$ & $\begin{array}{c}48,01 \text { * } \\
(35,1-65,6)\end{array}$ & $\begin{array}{c}42,86 \text { * } \\
(35,55-51,97)\end{array}$ & $\begin{array}{c}38,54 \text { * } \\
(32,98-5,04)\end{array}$ & $\begin{array}{c}29,09 \text { * } \\
(20,14-41,99)\end{array}$ & $\begin{array}{c}50,45 * \\
(35,98-70,74)\end{array}$ & $\begin{array}{c}40,24 \text { * } \\
(36,37-44,53)\end{array}$ \\
\hline $5-7$ & $\begin{array}{c}6,85 \text { * } \\
(5,55-8,28)\end{array}$ & $\begin{array}{c}7,15 \text { * } \\
(6,20-8,25)\end{array}$ & $\begin{array}{c}7,19 * \\
(6,41-8,06)\end{array}$ & $\begin{array}{c}6,77 \text { * } \\
(4,96-9,26)\end{array}$ & $\begin{array}{c}7,19 \text { * } \\
(5,82-8,87)\end{array}$ & $\begin{array}{c}7,04 \text { * } \\
(6,54-7,57)\end{array}$ \\
\hline $8-10$ & 1,00 & 1,00 & 1,00 & 1,00 & 1,00 & 1,00 \\
\hline \multicolumn{7}{|c|}{ Malformação congênita } \\
\hline Sim & $\begin{array}{c}30,69 * \\
(18,2-51,8)\end{array}$ & $\begin{array}{c}21,98 \text { * } \\
(15,81-30,55)\end{array}$ & $\begin{array}{c}31,29 * \\
(25,51-38,37)\end{array}$ & $\begin{array}{c}28,86 \text { * } \\
(16,25-51,25)\end{array}$ & $\begin{array}{c}32,98 \text { * } \\
(18,45-58,95)\end{array}$ & $\begin{array}{c}28,35 \text { * } \\
(24,35-33,01)\end{array}$ \\
\hline Não & 1,00 & 1,00 & 1,00 & 1,00 & 1,00 & 1,00 \\
\hline \multicolumn{7}{|l|}{ Gravidez e parto } \\
\hline \multicolumn{7}{|l|}{ Tipo de gravidez } \\
\hline Única & 1,00 & 1,00 & 1,00 & 1,00 & 1,00 & 1,00 \\
\hline Dupla/Múltipla & $\begin{array}{c}3,57 \text { * } \\
(2,60-4,89)\end{array}$ & $\begin{array}{c}4,35 \text { * } \\
(3,44-5,49)\end{array}$ & $\begin{array}{c}3,96 \text { * } \\
(3,29-4,76)\end{array}$ & $\begin{array}{c}5,42 \text { * } \\
(3,48-8,43)\end{array}$ & $\begin{array}{c}3,93 \text { * } \\
(2,85-5,40)\end{array}$ & $\begin{array}{c}4,08 \text { * } \\
(3,63-4,59)\end{array}$ \\
\hline \multicolumn{7}{|c|}{ Número de consultas de pré-natal } \\
\hline $0-3$ & $\begin{array}{c}4,33 \text { * } \\
(3,64-5,16)\end{array}$ & $\begin{array}{c}5,25 \text { * } \\
(4,58-6,01)\end{array}$ & $\begin{array}{c}7,80 * \\
(6,90-8,81)\end{array}$ & $\begin{array}{c}7,74 \text { * } \\
(5,53-10,84)\end{array}$ & $\begin{array}{c}5,21 * \\
(4,17-6,50)\end{array}$ & $\begin{array}{c}5,66 \text { * } \\
(5,27-6,07)\end{array}$ \\
\hline $4-6$ & $\begin{array}{c}1,84 \text { * } \\
(1,55,2-19)\end{array}$ & $\begin{array}{c}1,86 \text { * } \\
(1,34-2,12)\end{array}$ & $\begin{array}{c}3,55 \text { * } \\
(3,22-3,92)\end{array}$ & $\begin{array}{c}3,49 * \\
(2,66-4,57)\end{array}$ & $\begin{array}{c}3,31 \text { * } \\
(2,75-3,95)\end{array}$ & $\begin{array}{c}2,51 * \\
(2,35-2,67)\end{array}$ \\
\hline 7 e mais & 1,00 & 1,00 & 1,00 & 1,00 & 1,00 & 1,00 \\
\hline \multicolumn{7}{|l|}{ Tipo de parto } \\
\hline Cesáreo & $\begin{array}{c}0,83 \text { * } \\
(0,73-0,94)\end{array}$ & $\begin{array}{c}0,75 \text { * } \\
(0,68-0,83)\end{array}$ & $\begin{array}{c}0,88 \text { * } \\
(0,81-0,95)\end{array}$ & $\begin{array}{c}1,02 * * \\
(0,82-1,27)\end{array}$ & $\begin{array}{c}0,83 \text { * } \\
(0,71-0,98)\end{array}$ & $\begin{array}{c}0,84 \text { * } \\
(0,79-0,88)\end{array}$ \\
\hline Vaginal & 1,00 & 1,00 & 1,00 & 1,00 & 1,00 & 1,00 \\
\hline \multicolumn{7}{|l|}{ Assistência } \\
\hline \multicolumn{7}{|c|}{ Completude do serviço } \\
\hline Alta & 1,00 & 1,00 & 1,00 & 1,00 & 1,00 & 1,00 \\
\hline Intermediária & $\begin{array}{c}0,56 \text { * } \\
(0,47-0,67)\end{array}$ & $\begin{array}{c}0,51 * \\
(0,45-0,57)\end{array}$ & $\begin{array}{c}0,81 * \\
(0,74-0.88)\end{array}$ & $\begin{array}{c}0,45 \text { * } \\
(0,35-0,58)\end{array}$ & $\begin{array}{c}0,50 \text { * } \\
(0,42-0,60)\end{array}$ & $\begin{array}{c}0,65 \text { * } \\
(0,61-0,69)\end{array}$ \\
\hline Baixa & $\begin{array}{c}0,30 \text { * } \\
(0,20-0,37)\end{array}$ & $\begin{array}{c}0,31 \text { * } \\
(0,27-0,35)\end{array}$ & $\begin{array}{c}0,79 \text { * } \\
(0,69-0,90)\end{array}$ & $\begin{array}{c}0,61 \text { * } \\
(0,44-0,84)\end{array}$ & $\begin{array}{c}0,37 \text { * } \\
(0,30-0,46)\end{array}$ & $\begin{array}{c}0,46 \text { * } \\
(0,43-0,50)\end{array}$ \\
\hline
\end{tabular}

Fonte: elaborada pelos autores com base em dados do Sistema de Informações sobre Mortalidade e Sistema de Informações de Nascidos Vivos, Secretaria de Vigilância em Saúde, Ministério da Saúde.

Nota: valores da significância estatística (valor de $p$ ) do teste de qui-quadrado $\left(\chi^{2}\right)$ : * valor de $p<0,20$; ** valor de $p>0,20$. 


\section{Tabela 3}

Valores da odds ratio ajustada $\left(\mathrm{OR}_{\mathrm{aj}}\right)$, respectivos intervalos de $95 \%$ de confiança (IC95\%) e significância estatística (valor de p) resultantes do modelo multinível para associação entre a mortalidade infantil e os determinantes individuais e contextuais para o conjunto das 27 capitais e seus agregados nas respectivas macrorregiões, 2012.

\begin{tabular}{|c|c|c|c|c|c|c|}
\hline Variáveis & $\begin{array}{c}\text { Norte } \\
\mathrm{OR}_{\mathrm{aj}}(\mathrm{IC} 95 \%)\end{array}$ & $\begin{array}{c}\text { Nordeste } \\
\text { OR }_{\text {aj }}(I C 95 \%)\end{array}$ & $\begin{array}{c}\text { Sudeste } \\
\text { OR }_{\mathrm{aj}}(\mathrm{IC} 95 \%)\end{array}$ & $\begin{array}{c}\text { Sul } \\
\text { OR }_{\text {aj }}(I C 95 \%)\end{array}$ & $\begin{array}{l}\text { Centro-oeste } \\
\mathrm{OR}_{\mathrm{aj}}(\mathrm{IC} 95 \%)\end{array}$ & $\begin{array}{l}27 \text { capitais } \\
\text { OR }_{\text {aj }}(\text { IC95\%) }\end{array}$ \\
\hline \multicolumn{7}{|l|}{ Maternas } \\
\hline \multicolumn{7}{|l|}{ Idade da mãe (anos) } \\
\hline$<20$ & $\begin{array}{c}1,55 \text { * } \\
(1,11-2,15)\end{array}$ & $\begin{array}{c}1,54 \text { * } \\
(1,17-2,02)\end{array}$ & $\begin{array}{c}1,24 \text { * } \\
(1,01-1,54)\end{array}$ & & $\begin{array}{c}3,58 * \\
(2,02-6,34)\end{array}$ & $\begin{array}{c}1,43 \text { * } \\
(1,24-1,64)\end{array}$ \\
\hline $20-34$ & 1,00 & 1,00 & 1,00 & & 1,00 & 1,00 \\
\hline 35 e mais & $\begin{array}{c}1,17 * * \\
(0,70-1,76)\end{array}$ & $\begin{array}{c}1,06 \text { ** } \\
(0,79-1,41)\end{array}$ & $\begin{array}{c}0,91 * * \\
(0,74-1,13)\end{array}$ & & $\begin{array}{c}1,23 * * \\
(0,77-1,96)\end{array}$ & $\begin{array}{c}0,98 * * \\
(0,85-1,13)\end{array}$ \\
\hline \multicolumn{7}{|l|}{ Estado civil } \\
\hline Casada, união estável & 1,00 & & 1,00 & & & 1,00 \\
\hline Solteira, separada, viúva & $\begin{array}{c}1,25 * * \\
(0,95-1,63)\end{array}$ & & $\begin{array}{c}1,46 \text { * } \\
(1,24-1,72)\end{array}$ & & & $\begin{array}{c}1,24 \text { * } \\
(1,11-1,38)\end{array}$ \\
\hline \multicolumn{7}{|l|}{ Escolaridade (anos) } \\
\hline$<8$ & $\begin{array}{c}1,22 * * \\
(0,93-1,62)\end{array}$ & & & $\begin{array}{c}1,85 \text { * } \\
(1,09-3,12)\end{array}$ & & $\begin{array}{c}1,09 * * \\
(0,97-1,23)\end{array}$ \\
\hline 8 e mais & 1,00 & & & 1,00 & & 1,00 \\
\hline \multicolumn{7}{|l|}{ Ocupação } \\
\hline Com ocupação & 1,00 & 1,00 & 1,00 & & & 1,00 \\
\hline Sem ocupação & $\begin{array}{c}2,06 \text { * } \\
(1,14-4,37)\end{array}$ & $\begin{array}{c}2,83 \text { * } \\
(1,39-4,71)\end{array}$ & $\begin{array}{c}1,29 * * \\
(0,83-1,98)\end{array}$ & & & $\begin{array}{c}1,74 \text { * } \\
(1,19-2,45)\end{array}$ \\
\hline \multicolumn{7}{|l|}{ Raça/Cor } \\
\hline Branca & & 1,00 & 1,00 & & 1,00 & 1,00 \\
\hline Não branca & & $\begin{array}{c}1,44 \text { * } \\
(1,10-1,88)\end{array}$ & $\begin{array}{c}1,11 * * \\
(0,95-1,30)\end{array}$ & & $\begin{array}{c}1,47 \text { * } \\
(1,07-2,24)\end{array}$ & $\begin{array}{c}1,16 \text { * } \\
(1,03-1,31)\end{array}$ \\
\hline \multicolumn{7}{|l|}{ Número de filhos vivos } \\
\hline 0 & $\begin{array}{c}0,80 * * \\
(0,59-1,09)\end{array}$ & $\begin{array}{c}0,87 * * \\
(0,69-1,10)\end{array}$ & $\begin{array}{c}0,85 \text { * } \\
(0,71-0,98)\end{array}$ & & & $\begin{array}{c}0,86 \text { * } \\
(0,76-0,97)\end{array}$ \\
\hline 1 & 1,00 & 1,00 & 1,00 & & & 1,00 \\
\hline 2 e mais & $\begin{array}{c}1,14 \text { ** } \\
(0,83-1,55)\end{array}$ & $\begin{array}{c}1,37 \text { * } \\
(1,05-1,79)\end{array}$ & $\begin{array}{c}1,36 \text { * } \\
(1,11-1,68)\end{array}$ & & & $\begin{array}{c}1,28 \text { * } \\
(1,12-1,46)\end{array}$ \\
\hline \multicolumn{7}{|l|}{ Número de filhos mortos } \\
\hline 0 & & & & & 1,00 & \\
\hline 1 & & & & & $\begin{array}{c}0,90 * * \\
(0,54-1,50)\end{array}$ & \\
\hline 2 e mais & & & & & $\begin{array}{c}2,43 \text { * } \\
(1,16-5,11)\end{array}$ & \\
\hline \multicolumn{7}{|l|}{ Criança } \\
\hline \multicolumn{7}{|l|}{ Sexo } \\
\hline Feminino & 1,00 & & 1,00 & & 1,00 & 1,00 \\
\hline Masculino & $\begin{array}{c}1,25 * \\
(0,99-1,58)\end{array}$ & & $\begin{array}{c}1,12 * * \\
(0,96-1,29)\end{array}$ & & $\begin{array}{c}1,44 * \\
(1,01-2,05)\end{array}$ & $\begin{array}{c}1,15 \text { * } \\
(1,04-1,26)\end{array}$ \\
\hline \multicolumn{7}{|l|}{ Peso ao nascer } \\
\hline Muito baixo peso & $\begin{array}{c}46,12 \text { * } \\
(24,54-86,68)\end{array}$ & $\begin{array}{c}103,75 \text { * } \\
(65,53-164-26)\end{array}$ & $\begin{array}{c}76,27 \text { * } \\
(53,20-109,34)\end{array}$ & $\begin{array}{c}73,69 * \\
(27,91-134,55)\end{array}$ & $\begin{array}{c}212,77 \text { * } \\
(85,13-531,77)\end{array}$ & $\begin{array}{c}80,20 * \\
(63,36-101,53)\end{array}$ \\
\hline Baixo peso & $\begin{array}{c}5,27 \text { * } \\
(3,70-7,48)\end{array}$ & $\begin{array}{c}5,36 \text { * } \\
(4,02-7,14)\end{array}$ & $\begin{array}{c}4,82 \text { * } \\
(3,90-5,97)\end{array}$ & $\begin{array}{c}7,52 \text { * } \\
(3,91-14,46)\end{array}$ & $\begin{array}{c}9,99 * \\
(5,79-17,25)\end{array}$ & $\begin{array}{c}5,26 \text { * } \\
(4,57-9,92)\end{array}$ \\
\hline Normal & 1,00 & 1,00 & 1,00 & 1,00 & 1,00 & 1,00 \\
\hline
\end{tabular}

(continua) 
Tabela 3 (continuação)

\begin{tabular}{|c|c|c|c|c|c|c|}
\hline Variáveis & $\begin{array}{c}\text { Norte } \\
\text { OR }_{\text {aj }}(\text { IC95\%) }\end{array}$ & $\begin{array}{c}\text { Nordeste } \\
\mathrm{OR}_{\mathrm{aj}}(\mathrm{IC} 95 \%)\end{array}$ & $\begin{array}{c}\text { Sudeste } \\
\mathrm{OR}_{\mathrm{aj}}(\mathrm{IC} 95 \%)\end{array}$ & $\begin{array}{c}\text { Sul } \\
\text { OR }_{\text {aj }}(\mathrm{IC} 95 \%)\end{array}$ & $\begin{array}{l}\text { Centro-oeste } \\
\text { OR }_{\mathrm{aj}}(\mathrm{IC} 95 \%)\end{array}$ & $\begin{array}{c}27 \text { capitais } \\
\mathrm{OR}_{\mathrm{aj}}(\mathrm{IC95 \%} \%)\end{array}$ \\
\hline \multicolumn{7}{|l|}{ Criança } \\
\hline \multicolumn{7}{|c|}{ Idade gestacional (semanas) } \\
\hline$>37$ & $\begin{array}{c}2,29 * \\
(1,03-3,78)\end{array}$ & $\begin{array}{c}3,77 \text { * } \\
(1,59-5,21)\end{array}$ & $\begin{array}{c}8,62 \text { * } \\
(5,36-11,17)\end{array}$ & $\begin{array}{c}13,83 \text { * } \\
(10,41-18,49)\end{array}$ & $\begin{array}{c}6,64 \text { * } \\
(5,56-7,92)\end{array}$ & $\begin{array}{c}7,52 \text { * } \\
(5,56-9,92)\end{array}$ \\
\hline 37 e mais & 1,00 & 1,00 & 1,00 & 1,00 & 1,00 & 1,00 \\
\hline \multicolumn{7}{|c|}{ Apgar no $1 \underline{0}$ minuto } \\
\hline $0-4$ & $\begin{array}{c}14,83 \text { * } \\
(8,84-24,88)\end{array}$ & $\begin{array}{c}13,09 \text { * } \\
(9,54-17,96)\end{array}$ & $\begin{array}{c}10,72 \text { * } \\
(8,24-13,93)\end{array}$ & $\begin{array}{c}5,81 \text { * } \\
(2,85-11,88)\end{array}$ & $\begin{array}{c}18,70 \text { * } \\
(9,21-380,1)\end{array}$ & $\begin{array}{c}11,54 \text { * } \\
(9,70-13,72)\end{array}$ \\
\hline $5-7$ & $\begin{array}{c}3,97 \text { * } \\
(2,84-5,56)\end{array}$ & $\begin{array}{c}2,97 \text { * } \\
(2,33-3,78)\end{array}$ & $\begin{array}{c}2,50 * \\
(2,07-3,03)\end{array}$ & $\begin{array}{c}2,31 * \\
(1,31-4,04)\end{array}$ & $\begin{array}{c}2,98 * \\
(1,86-4,75)\end{array}$ & $\begin{array}{c}2,82 \text { * } \\
(2,48-3,19)\end{array}$ \\
\hline 8-10 & 1,00 & 1,00 & 1,00 & 1,00 & 1,00 & 1,00 \\
\hline \multicolumn{7}{|c|}{ Malformação congênita } \\
\hline Sim & $\begin{array}{c}27,51 * \\
(12,62-59,93)\end{array}$ & $\begin{array}{c}27,74 \text { * } \\
(15,97-48,18)\end{array}$ & $\begin{array}{c}37,48 \text { * } \\
(27,87-50,42)\end{array}$ & $\begin{array}{c}33,42 \text { * } \\
(13,56-62,39)\end{array}$ & $\begin{array}{c}21,80 \text { * } \\
(7,94-39,81)\end{array}$ & $\begin{array}{c}35,61 \text { * } \\
(28,24-44,90)\end{array}$ \\
\hline Não & 1,00 & 1,00 & 1,00 & 1,00 & 1,00 & 1,00 \\
\hline \multicolumn{7}{|l|}{ Gravidez e parto } \\
\hline \multicolumn{7}{|l|}{ Tipo de gravidez } \\
\hline Única & & & 1,00 & & & 1,00 \\
\hline Dupla/Múltipla & & & $\begin{array}{c}1,33 * * \\
(0,91,1,93)\end{array}$ & & & $\begin{array}{c}1,07 * * \\
(0,82-1,40)\end{array}$ \\
\hline \multicolumn{7}{|c|}{ Número de consultas de pré-natal } \\
\hline $0-3$ & $\begin{array}{c}1,70 * \\
(1,22-2,37)\end{array}$ & $\begin{array}{c}1,43 \text { * } \\
(1,07-1,91)\end{array}$ & $\begin{array}{c}1,76 \text { * } \\
(1,38-2,25)\end{array}$ & $\begin{array}{c}3,32 \text { * } \\
(1,73-6,35)\end{array}$ & $\begin{array}{c}2,89 * \\
(1,67-4,99)\end{array}$ & $\begin{array}{c}1,80 \text { * } \\
(1,55-2,09)\end{array}$ \\
\hline $4-6$ & $\begin{array}{c}1,17 \text { * } \\
(1,08-1,57)\end{array}$ & $\begin{array}{c}1,02 * * \\
(0,81-1,28)\end{array}$ & $\begin{array}{c}1,37 \text { * } \\
(1,15-1,63)\end{array}$ & $\begin{array}{c}1,38 \text { * } \\
(1,06-2,47)\end{array}$ & $\begin{array}{c}1,93 \text { * } \\
(1,26-2,97)\end{array}$ & $\begin{array}{c}1,28 \text { * } \\
(1,14-1,44)\end{array}$ \\
\hline 7 e mais & 1,00 & 1,00 & 1,00 & & 1,00 & 1,00 \\
\hline \multicolumn{7}{|c|}{ Assistência } \\
\hline \multicolumn{7}{|c|}{ Completude do serviço } \\
\hline Alta & & & & & 1,00 & \\
\hline Intermediária & & & & & $\begin{array}{c}0,27 \text { * } \\
(0,06-0,93)\end{array}$ & \\
\hline Baixa & & & & & $\begin{array}{c}0,19 \text { * } \\
(0,04-0,87)\end{array}$ & \\
\hline
\end{tabular}

AIC: critério de Akaike; BIC: critério de informação Bayesiano.

Fonte: elaborada pelos autores com base em dados do Sistema de Informações sobre Mortalidade e Sistema de Informações de Nascidos Vivos, Secretaria de Vigilância em Saúde, Ministério da Saúde.

Notas: Valores da significância estatística (valor de $p$ ): * valor de $p<0,010$; ** valor de $p>0,010$.

Número de unidades - nível 1: (Norte - 4.995; Nordeste - 8.693; Sudeste - 12.751; Sul - 1.791; Centro-oeste - 3.525; Capitais - 31.755); nível 2: (Norte 84; Nordeste - 2.173; Sudeste - 275; Sul - 52; Centro-oeste - 2.118; Capitais -702); nível 3: (Norte - 7; Nordeste - 9; Sudeste - 4; Sul - 3; Centro-oeste - 4; Capitais - 27).

Ajuste do modelo: Norte (AIC 1.951, 819 / BIC 2.088, 866); Nordeste (AIC 3.158, 71/BIC 3.297, 145); Sudeste (AIC 5.162, 108/BIC 5.309, 04; Sul (AIC 671, 707/BIC 757, 3.961); Centro-oeste (AIC 1.058, 492/BIC 1.172, 759); Capitais (AIC 12.099, 76/ BIC 12.273, 16).

Variâncias - nível 2 (ajustado pelo estabelecimento de saúde) e nível 3 (ajustado pelo estabelecimento de saúde e pela capital) Norte - nível 2: 0,11654487 (0,07966821)/Nível 3: 0,39564475 (02596284)/Nordeste - nível 2: 0,42584847 (0,1324203); nível 3: 0,55863323 (0,30268624); Sudeste - nível 2: 0,10882201 (0,0440365); nível 3: 0,00248253 (0,01431209); Sul - nível 2: 0,03995209 (0,13437628); nível 3: 3,6044215 (3,5387643); Centro-oeste - nível 2: 2,3135363 (0,73140467); nível 3: 6,0256696 (6,7930071); Capitais - nível 2: 0,26936676 (0,05132081; nível 3: variância: 1,1229798 (0,40503521). 
Os fatores relativos às condições do recém-nascido foram os que se apresentaram mais fortemente associados ao óbito. Destacam-se o baixo peso ao nascer, a prematuridade, a asfixia grave/moderada e a presença de malformações congênitas, expressando elevados valores da OR e compondo todos os modelos finais. Os recém-nascidos do sexo masculino expressaram maior chance de apresentar o desfecho entre os casos, exceto nas regiões Nordeste $(\mathrm{OR}=1,25)$ e Sul $(\mathrm{OR}=1,44)$ (Tabela 3$)$.

No que concerne à gestação e ao parto, o baixo número de consultas de pré-natal foi o fator mais fortemente associado ao óbito infantil, integrando o modelo de risco em todas as regiões. As gestações duplas/múltiplas compuseram o modelo de risco no conjunto das capitais e no Sudeste (Tabela 3).

$\mathrm{Na}$ análise da variável contextual, observou-se que a completude permaneceu no modelo final apenas nas capitais do Sudeste. Nessa região, os nascimentos em estabelecimentos de baixa e intermediária completude representaram fator de proteção ao óbito infantil, com valores da OR de 0,19 e 0,27 , respectivamente (Tabela 3 ).

A análise de interação evidenciou que o baixo número de consultas de pré-natal esteve relacionado ao óbito infantil independentemente do nível de completude do serviço nas capitais do Norte, Nordeste, Sudeste e Centro-oeste. Entretanto, no Sul verificou-se que os nascidos vivos de mães que realizaram entre quatro e seis consultas de pré-natal e nasceram em serviços de baixa completude apresentaram maior risco para a mortalidade infantil $(\mathrm{OR}=4,96)$ (Tabela 4).

Quanto à renda, a interação aponta para um efeito protetor para os nascidos vivos de mães com baixo número de consultas de pré-natal e que residem em cidades de baixa renda média. Nessas cidades, dentre os nascidos de mães que realizaram até três consultas a OR ajustada foi de 0,68, e dentre aquelas com quatro a seis consultas a OR foi de 0,54 (Tabela 4).

\section{Tabela 4}

Estimativas da odds ratio ajustada $\left(\mathrm{OR}_{\mathrm{aj}}\right)$, respectivos intervalos de $95 \%$ de confiança (IC95\%) e significância estatística (valor de p) resultantes do modelo multinível para interação entre o número de consultas de pré-natal e as variáveis contextuais (completude do estabelecimento de nascimento das crianças e Renda da capital de residência) para o conjunto das 27 capitais e seus agregados nas respectivas macrorregiões, 2012.

\begin{tabular}{|c|c|c|c|c|c|c|}
\hline Variáveis & $\begin{array}{c}\text { Norte } \\
\text { OR }_{\text {aj }} \\
(I C 95 \%)\end{array}$ & $\begin{array}{c}\text { Nordeste } \\
\text { OR }_{\text {aj }} \\
(I C 95 \%)\end{array}$ & $\begin{array}{l}\text { Sudeste } \\
\text { OR }_{\text {aj }} \\
(I C 95 \%)\end{array}$ & $\begin{array}{c}\text { Sul } \\
\text { OR }_{\text {aj }} \\
(I C 95 \%)\end{array}$ & $\begin{array}{c}\text { Centro-oeste } \\
\text { OR }_{\mathrm{aj}} \\
(\mathrm{IC} 95 \%)\end{array}$ & $\begin{array}{c}27 \text { capitais } \\
\text { OR }_{\mathrm{aj}} \\
(\mathrm{IC} 95 \%)\end{array}$ \\
\hline \multicolumn{7}{|c|}{ Consultas de pré-natal X completude } \\
\hline \multicolumn{7}{|l|}{ Modelo sem interação (consultas) } \\
\hline $0-3$ & $\begin{array}{c}4,06 \text { * } \\
(2,62-6,29)\end{array}$ & $\begin{array}{c}4,55 * \\
(3,59-5,77)\end{array}$ & $\begin{array}{c}7,20 * \\
(5,91-8,83)\end{array}$ & $\begin{array}{c}7,97 * \\
(5,06-12,56)\end{array}$ & $\begin{array}{c}5,27 * \\
(3,57-7,77)\end{array}$ & $\begin{array}{c}6,12 \text { * } \\
(5,38-6,97)\end{array}$ \\
\hline $4-6$ & $\begin{array}{c}2,08 * \\
(2,36-13,18)\end{array}$ & $\begin{array}{c}1,91 * \\
(1,52-2,39)\end{array}$ & $\begin{array}{c}3,40 * \\
(2,89-4,02)\end{array}$ & $\begin{array}{c}2,87 * \\
(1,89-4,35)\end{array}$ & $\begin{array}{c}3,27 * \\
(3,34-4,59)\end{array}$ & $\begin{array}{c}2,83 * \\
(2,53-3,18)\end{array}$ \\
\hline 7 e mais & 1,00 & 1,00 & 1,00 & 1,00 & 1,00 & 1,00 \\
\hline Completude baixa & $\begin{array}{c}0,45 * \\
(0,24-0,83)\end{array}$ & $\begin{array}{c}0,49 * \\
(0,33-0,72)\end{array}$ & $\begin{array}{c}0,81 * \\
(0,59-0,98)\end{array}$ & $\begin{array}{c}0,48 * * \\
(0,17-1,40)\end{array}$ & $\begin{array}{c}0,36 \text { * } \\
(0,19-0,67)\end{array}$ & $\begin{array}{c}0,52 \text { * } \\
(0,41-0,65)\end{array}$ \\
\hline Completude intermediária & $\begin{array}{c}0,62 * * \\
(0,33-1,15)\end{array}$ & $\begin{array}{c}0,66 \text { * } \\
(0,44-0,99)\end{array}$ & $\begin{array}{c}0,80 * \\
(0,59-0,98)\end{array}$ & $\begin{array}{c}0,67 * * \\
(0,29-1,57)\end{array}$ & $\begin{array}{c}0,51 * \\
(0,27-0,95)\end{array}$ & $\begin{array}{c}0,66 \text { * } \\
(0,53-0,81)\end{array}$ \\
\hline Completude alta & 1,00 & 1,00 & 1,00 & 1,00 & 1,00 & 1,00 \\
\hline \multicolumn{7}{|l|}{ Modelo com interação (consultas) } \\
\hline $0-3 \times$ completude baixa & $\begin{array}{c}0,72 * \star \\
(0,41-1,28)\end{array}$ & $\begin{array}{c}1,09 * * \\
(0,73-1,62)\end{array}$ & $\begin{array}{c}1,48 * * \\
(0,92-2,38)\end{array}$ & $\begin{array}{c}2,90 * * \\
(0,38-22,22)\end{array}$ & $\begin{array}{c}0,85 * \star \\
(0,44-1,65)\end{array}$ & $\begin{array}{c}0,86 * * \\
(0,68-1,08)\end{array}$ \\
\hline intermediária & $\begin{array}{c}1,12 * * \\
(0,67-1,86)\end{array}$ & $\begin{array}{c}1,32 \star * \\
(0,92-1,90)\end{array}$ & $\begin{array}{c}1,25 * * \\
(0,95-1,66)\end{array}$ & $\begin{array}{c}1,08 * * \\
(0,36-3,23)\end{array}$ & $\begin{array}{c}1,17 * \star \\
(0,67-2,03)\end{array}$ & $\begin{array}{c}1,17 * * \\
(0,98-1,40)\end{array}$ \\
\hline 4-6 X completude baixa & $\begin{array}{c}0,79 * * \\
(0,47-1,35)\end{array}$ & $\begin{array}{c}0,86 * * \\
(0,60-1,25)\end{array}$ & $\begin{array}{c}1,10 * * \\
(0,79-1,52)\end{array}$ & $\begin{array}{c}4,96 * \\
(1,79-13,70)\end{array}$ & $\begin{array}{c}1,09 * * \\
(0,62-1,93)\end{array}$ & $\begin{array}{c}0,85 * * \\
(0,70-1,03)\end{array}$ \\
\hline 4-6 X completude intermediária & $\begin{array}{c}0,84 * \star \\
(, 0,51-1,38)\end{array}$ & $\begin{array}{c}1,18 * * \\
(0,85-1,10)\end{array}$ & $\begin{array}{c}1,11 * * \\
(0,88-1,39)\end{array}$ & $\begin{array}{c}1,55 * * \\
(0,78-3,08)\end{array}$ & $\begin{array}{c}1,03 * * \\
(0,65-, 63)\end{array}$ & $\begin{array}{c}0,06 * * \\
(0,90-1,24)\end{array}$ \\
\hline
\end{tabular}

(continua) 
Tabela 4 (continuação)

\begin{tabular}{|c|c|c|c|c|c|c|}
\hline Variáveis & $\begin{array}{c}\text { Norte } \\
\text { OR }_{\mathrm{aj}} \\
(\mathrm{IC} 95 \%)\end{array}$ & $\begin{array}{c}\text { Nordeste } \\
\text { OR }_{\mathrm{aj}} \\
(\mathrm{IC} 95 \%)\end{array}$ & $\begin{array}{l}\text { Sudeste } \\
\text { OR }_{\mathrm{aj}} \\
(\mathrm{IC} 95 \%)\end{array}$ & $\begin{array}{c}\text { Sul } \\
\text { OR }_{\text {aj }} \\
(I C 95 \%)\end{array}$ & $\begin{array}{c}\text { Centro-oeste } \\
\text { OR }_{\mathrm{aj}} \\
(\mathrm{IC} 95 \%)\end{array}$ & $\begin{array}{c}27 \text { capitais } \\
\text { OR }_{\text {aj }} \\
(I C 95 \%)\end{array}$ \\
\hline \multicolumn{7}{|c|}{ Consultas de pré-natal $\mathrm{X}$ renda } \\
\hline \multicolumn{7}{|c|}{ Modelo sem interação (consultas) } \\
\hline $0-3$ & & & & & & $\begin{array}{c}6,72 \text { * } \\
(5,27-8,59)\end{array}$ \\
\hline $4-6$ & & & & & & $\begin{array}{c}3,40 * \\
(2,77-4,18)\end{array}$ \\
\hline 7 e mais & & & & & & 1,00 \\
\hline Renda baixa & & & & & & $\begin{array}{c}0,83 * * \\
(0,63-1,11)\end{array}$ \\
\hline Renda intermediária & & & & & & $\begin{array}{c}0,84 * * \\
(0,63-1,13)\end{array}$ \\
\hline Renda alta & & & & & & 1,00 \\
\hline \multicolumn{7}{|c|}{ Modelo com interação (consultas) } \\
\hline 0-3 X renda baixa & & & & & & $\begin{array}{c}0,68 \text { * } \\
(0,52-0,90)\end{array}$ \\
\hline 0-3 X renda intermediária & & & & & & $\begin{array}{c}1,18 \text { ** } \\
(0,90-1,56)\end{array}$ \\
\hline $4-6 \times$ renda baixa & & & & & & $\begin{array}{c}0,54 \text { * } \\
(0,43-0,69)\end{array}$ \\
\hline 4-6 X renda intermediária & & & & & & $\begin{array}{c}1,05 * * \\
(0,83-1,32)\end{array}$ \\
\hline
\end{tabular}

Fonte: elaborada pelos autores com base em dados do Sistema de Informações sobre Mortalidade e Sistema de Informações de Nascidos Vivos, Secretaria de Vigilância em Saúde, Ministério da Saúde.

Notas: valores da significância estatística: * valor de $p<0,05 ; * *$ valor de $p>0,05$.

Variâncias da interação entre número de consultas de pré-natal e completude ajustadas pelo estabelecimento de saúde (nível 2) e ajustadas pelo estabelecimento de saúde e pela capital (nível 3): Norte - nível 2: 0,16627451 (0,650506); nível 3: 1,12030019 (7,70300011); Nordeste -

nível 2: 0,58701576 (0,12291005); nível 3: 0,2375024 (0,03614717); Sudeste - nível 2: 0,24244059 (0,04379712); nível 3: 1,0410015 (3,27900009);

Sul - nível 2: 0,8774731 (0,38175092); nível 3: 6,38400013 (3,45300007); Centro-oeste - nível 2: 0,50065335(0,14532047); nível 3: 6,25300013

(3,61200007); capitais - nível 2: 0,42015577(0,04725237); nível 3: 0,01274927 (0,01679953).

Variâncias da interação entre número de consultas de pré-natal e renda: nível 2 (ajustado pelo estabelecimento de saúde); variância: 0,45962406

(0.04973229; nível 3: 0,01212106 (0,01530843)

\section{Discussão}

A composição dos modelos finais resultante desta pesquisa expressa um predomínio dos fatores biológicos na determinação das mortes infantis. Os achados evidenciam ainda o peso da assistência materno-infantil na determinação dos óbitos de menores de um ano e a importância dos determinantes sociais, inclusive na mediação do acesso e da qualidade à atenção à saúde.

O baixo peso ao nascer é o fator individual de maior influência na saúde e sobrevivência do recém-nascido, apresentando uma relação intrínseca com a prematuridade 22. Essas condições, em geral, estão associadas a extremos de idade materna, presença de malformações congênitas, história prévia de natimorto, tabagismo na gravidez, baixa escolaridade da mãe, ganho de peso materno insuficiente, hipertensão arterial, sangramento vaginal, infecção do trato geniturinário e baixa qualidade da assistência pré-natal 22,23.

A presença de anomalias congênitas demonstrou forte associação com a mortalidade infantil nas capitais brasileiras. Achados que ratificam a importância da detecção das malformações no pré-natal e a garantia do acompanhamento especializado, da programação do parto e do atendimento ao recém-nascido como estratégias para reduzir as mortes por essa causa 24. 
Ressalta-se também a associação entre a asfixia no 1ํ minuto e o óbito infantil em todos as regiões analisadas. $\mathrm{O}$ que assevera que índices de Apgar menores ou iguais a oito colocam o recém-nascido em situação de maior vulnerabilidade 11,25.

A relação entre as mortes infantis e o sexo masculino observada nos achados desta pesquisa é também relatada por outros autores 18 que a atribuem ao amadurecimento do pulmão que ocorre mais tardiamente neste grupo, resultando em uma maior incidência de problemas respiratórios e maior frequência de hospitalização.

Apesar da preponderância dos fatores biológicos, cabe destacar que estes podem resultar das características socioeconômicas das mães 25,26,27. Os achados deste estudo demonstraram que, em geral, ser filho de mães nos extremos etários (adolescentes ou com 35 anos e mais), solteira, separada ou viúva, de baixa escolaridade, sem ocupação remunerada e não brancas consistiu em fator de risco para a mortalidade de menores de um ano na maioria dos modelos, reiterando achados de pesquisas anteriores $27,28,29$.

Tais resultados expressam a vulnerabilidade social em que se inserem essas mulheres, considerando a relação entre a escolaridade, o acesso ao emprego e à idade materna. Segundo o Instituto Brasileiro de Geografia e Estatística (IBGE) 30, a maternidade é a principal responsável pelo afastamento das mulheres da escola. A pesquisa registrou que apenas 28,5\% das mães com idades entre 15 e 17 anos estudavam, e entre as mulheres que tiveram filhos com idades entre 18 e 24 anos apenas $10 \%$ permaneciam estudando. Esses dados evidenciam a dificuldade de conciliar a maternidade com o estudo e, consequentemente, os entraves vivenciados por essas mães para o ingresso ou retorno ao mercado de trabalho 30 .

A multiparidade consistiu em fator de risco na maior parte das capitais brasileiras. A alta paridade em geral está relacionada, sobretudo nos países em desenvolvimento, ao baixo nível socioeconômico, às desigualdades de oportunidades educacionais, ao acesso e utilização de métodos contraceptivos para o planejamento familiar, tornando as mulheres desse grupo mais vulneráveis a resultados adversos em suas gestações 31 .

Por outro lado, os nascimentos de mães primíparas consistiram em fator de proteção para o óbito infantil. Achados que coadunam com o contexto demográfico de redução da fecundidade e postergação da maternidade verificado no país 32 . Lima 33 ressalta que primíparas com idade materna avançada tendem a demonstrar características positivas de conduta com a saúde pré-natal, como a suspensão do uso de tabaco e álcool, adoção de dieta balanceada, e as maiores chances de condições socioeconômicas e psicológicas favoráveis, habilitando estas mães a experimentarem gravidezes com resultados obstétricos favoráveis.

Os resultados da pesquisa evidenciam também a importância dos fatores relacionados à atenção à saúde materno e infantil, especialmente à frequência às consultas de pré-natal, que figurou como fator de risco presente em todos os modelos finais aqui apresentados. Essa relação tem sido demonstrada na literatura 7,11,18,25,31 como uma das variáveis mais importantes relacionadas à gestação e ao parto na prevenção da morbimortalidade infantil.

A assistência pré-natal tem um papel fundamental na proteção da vida e da saúde da gestante e do recém-nascido, uma vez que, garantido o acesso e a qualidade dos cuidados pré-natais, eles podem contornar problemas obstétricos, prevenir danos, reduzir a ocorrência de prematuridade e do baixo peso ao nascer, além de assegurar partos e nascimentos saudáveis 34,35. Em países com baixas taxas de mortalidade infantil, a atenção pré-natal de boa qualidade foi um dos mais importantes investimentos para a redução destas mortes, sobretudo no componente neonatal, mesmo entre grupos populacionais com condições socioeconômicas mais desfavoráveis 28 .

O tipo de gravidez só permaneceu no modelo final do conjunto das capitais brasileiras e no Sudeste. A associação entre gravidez múltipla e óbito infantil pode ser influenciada pela prematuridade e pelo baixo peso ao nascer, muito prevalentes entre os gemelares 36 .

Neste estudo, o tipo de parto foi a única variável a não permanecer em nenhum dos seis modelos de risco para a mortalidade infantil nas capitais brasileiras, de modo semelhante ao verificado em outras pesquisas no país, nas quais não observou-se associação significante entre a via de parto e o óbito infantil 18,25,26.

O parto operatório, quando corretamente indicado, a exemplo das gestações de alto risco, de sofrimento fetal, de falta de progresso no trabalho de parto e de pré-eclâmpsia é considerado um procedi- 
mento relevante para a redução dos riscos perinatais, aumentando a sobrevida dos recém-nascidos 37 . Entretanto, a literatura sugere a existência do componente socioeconômico na determinação da via de parto, verificando-se maiores taxas de cesarianas em hospitais privados e em mulheres de maior renda familiar 38 .

A completude do serviço de nascimento das crianças integrou o modelo de risco apenas das capitais da Região Sudeste, onde os nascimentos ocorridos em serviços de baixa e intermediária completude representaram fator de proteção para o óbito infantil.

A rede hospitalar para a assistência materna e neonatal do Sistema Único de Saúde (SUS) é predominantemente de pequeno porte, baixa complexidade, com insuficiente desempenho das práticas assistenciais ao parto e baixo desenvolvimento das práticas recomendáveis e epidemiológicas 19 .

Tais considerações permitem pressupor que o fator protetor para os serviços de baixa/intermediária completude observado no presente estudo pode estar relacionado ao fato de que as unidades de alta complexidade costumam se caracterizar por uma maior demanda de gestantes e recém-nascidos de maior risco social e com piores condições de saúde 11. Estudos realizados no país referem maior vulnerabilidade socioeconômica para as mulheres que deram à luz nos hospitais do SUS, verificando que estas mães são proporcionalmente mais jovens, sem companheiro, menos escolarizadas, com menor nível socioeconômico e têm menos acesso ao pré-natal 39,40.

Nesse sentido, a análise de interação desenvolvida neste estudo entre o número de consultas de pré-natal e a completude apresenta importantes elementos para a compreensão das inter-relações entre os fatores implicados na determinação do óbito infantil. Os resultados demonstram que a realização de um baixo número de atendimentos é, incontestavelmente, fator de risco para o óbito infantil. Porém, essa variável ao interagir com a completude, nas capitais da Região Sul, revelou que ter realizado baixo número de consultas pré-natais e nascer em serviços de baixa completude representaram um risco relativamente maior quando comparado às unidades de alta completude.

Entretanto, cabe ainda destacar diferenças importantes, e até mesmo simbólicas, entre os resultados observados nas demais regiões, nas quais a realização de um insuficiente número de consultas de pré-natal foi fator de risco para a mortalidade infantil independentemente da completude da unidade de nascimento. Esses achados levam a postular sobre uma possível "homogeneidade" em níveis baixos de qualidade da assistência à saúde.

Nessa perspectiva, pesquisas realizadas por Polgliane et al. 28 na cidade de Vitória, Espírito Santo, e por Leal et al. 41 nos 252 municípios prioritários para a redução da mortalidade infantil na Amazônia Legal e Nordeste, apontam para níveis muito baixos de adequação da atenção ao pré-natal. Em Vitória, no período de 1999 a 2010, o processo de assistência pré-natal foi inadequado para a totalidade das gestantes segundo os padrões da Organização Mundial da Saúde (OMS), e para mais de 95\% das gestantes, segundo os parâmetros do Programa de Humanização no Pré-natal e Nascimento (PHPN), levando à conclusão de que a quantidade de consultas e a frequência de realização dos procedimentos não asseguram a adequação da assistência prestada.

$\mathrm{Na}$ análise da interação entre as consultas pré-natais e a renda da cidade, os achados apontam para uma relevante e aparentemente paradoxal situação, na qual ter realizado um baixo número de consultas pré-natais e nascer em cidades de alta renda média representaram risco relativamente maior quando comparado aos que nasceram em capitais de baixa renda per capita. Estudo realizado em Recife, Pernambuco, detectou uma relação inversa entre a condição de vida e a magnitude da mortalidade infantil, revelando desigualdades encobertas nos indicadores médios de saúde 42 .

Pode-se inferir por meio desses resultados que as capitais mais desenvolvidas economicamente podem ocultar graves disparidades sociais, representando altos níveis de privação e até mesmo exclusão das parcelas mais pobres da população. Estudos confirmam que países com alto grau de iniquidades de renda, mesmo grupos populacionais de renda média, apresentam uma situação de saúde pior do que grupos de renda inferior, mas que vivem em uma sociedade mais equitativa 43 .

De modo geral, os resultados demonstram que, embora se verifique um certo consenso entre os fatores determinantes da mortalidade infantil entre as capitais, alguns diferenciais regionais são evidenciados. Se por um lado os fatores biológicos apresentaram certa homogeneidade na composição dos modelos finais de risco para a mortalidade infantil, por outro é possível identificar maior relevância das características maternas como expressão das condições socioeconômicas das gestantes e dos recém-nascidos, nas regiões Norte, Nordeste e Sudeste, quando comparadas ao centro-sul do país. 
Vale considerar que esse quadro de discrepâncias regionais retratado na análise das capitais brasileiras ainda abriga diferenciais intraurbanos dentro de cada cidade, aqui não tratados, produzindo variados níveis de risco em distintos subgrupos populacionais.

Ressalta-se ainda que tanto o acesso ao pré-natal como a qualidade da assistência prestada são influenciados pela condição socioeconômica da mulher, constatando-se um cenário de iniquidades no qual as gestantes do Norte e do Nordeste brasileiro e de baixa condição social realizam menor número de consultas e de pior qualidade, e as mulheres pertencentes às classes mais privilegiadas podem ser mais saudáveis durante a gravidez por utilizar os serviços antenatais, enfim, ter mais recursos para gerar e manter os filhos mais saudáveis 28,41 .

Os modelos explicativos sobre os determinantes da mortalidade infantil vão ao encontro da tese da importância de ações intersetoriais na busca de uma melhor condição de saúde para as populações. Mesmo ficando claro o resultado dos esforços empreendidos pelo setor saúde, é inquestionável a necessidade da parceria dos demais setores na construção de uma sociedade mais equânime e saudável 44.

Por fim, ressalta-se a relevância da utilização do método multinível no estudo dos determinantes da mortalidade infantil, por permitir considerar o contexto e a relação entre as exposições intra e entre grupos. Zanini et al. 11 afirmam que modelos multiníveis não necessariamente produzirão resultados diferentes dos procedimentos tradicionais de análise, mas as estimativas tendem a ser mais acuradas, sendo capaz de capturar efeitos significativos em cada nível. Ademais, possibilitam o aprofundamento da compreensão sobre os determinantes sociais e fundamentam o planejamento das intervenções de saúde pública e das decisões políticas 45 .

Entre as limitações da pesquisa registram-se possíveis vieses de aferição em função da qualidade das informações pelo uso de dados secundários e a impossibilidade de inclusão de outros possíveis determinantes da mortalidade infantil, o que circunscreveu esta análise às variáveis preditoras presentes no SINASC.

\section{Colaboradores}

L. T. S. Maia contribuiu com a concepção e planejamento, tabulação, processamento, análise e interpretação dos dados, redação, revisão crítica do conteúdo e aprovação da versão final do manuscrito. W. V. Souza contribuiu na concepção e planejamento, análise e interpretação dos dados, revisão crítica do conteúdo e aprovação da versão final do manuscrito. A. C. G. Mendes contribuiu com a concepção e planejamento, interpretação dos dados, revisão crítica do conteúdo, aprovação da versão final do manuscrito.

\section{Informações adicionais}

ORCID: Lívia Teixeira de Souza Maia (0000-00020161-7729); Wayner Vieira de Souza (0000-00020939-9332); Antonio da Cruz Gouveia Mendes (0000-0002-3381-134X).

\section{Agradecimentos}

À Fundação de Amparo à Ciência e Tecnologia do Estado de Pernambuco (FACEPE) e ao Ministério da Saúde.

\section{Referências}

1. França E, Lansky S. Mortalidade infantil neonatal no Brasil: situação, tendências e perspectivas. In: Rede Interagencial de Informações para Saúde, organizador. Demografia e saúde: contribuição para análise de situação e tendências. Brasília: Organização Pan-Americana da Saúde, 2009. p. 83-112. (Série G. Estatística e Informação em Saúde) (Série Informe de Situação e Tendências).

2. Mombelli MA, Sass A, Molena CAF, Téston EF, Marcon SS. Fatores de risco para mortalidade infantil em municípios do Estado do Paraná, de 1997 a 2008. Rev Paul Pediatr 2012; 30:187-94.

3. Barbosa TAGS, Coelho KR, Andrade GN, Bittencourt SDA, Leal MC, Gazzinelli A. Determinantes da mortalidade infantil em municípios do Vale do Jequitinhonha, MG, Brasil. REME Rev Min Enferm 2014; 18:907-22.

4. Viellas, EF, Domingues RMSM, Dias MAB, Gama SGN, Theme Filha MM, Costa JV, et al. Assistência pré-natal no Brasil. Cad Saúde Pública 2014; 30 Suppl:S85-100.

5. Cunha SF, D’Eça Júnior A, Rios ATF, Pestana AL, Mochel EG, Paiva SS. Peregrinação no anteparto em São Luís - MA. Cogitare Enferm 2010; 15:441-7. 
6. Mosley WH, Chen LC. An analytical framework for the study of child survival in developing countries. Popul Dev Rev 1984; 10 Suppl:25-45.

7. Leal MC, Bittencourt SDA, Torres RMC, Niquini RP, Souza Jr. PRB. Determinantes do óbito infantil no Vale do Jequitinhonha e nas regiões Norte e Nordeste do Brasil. Rev Saúde Pública 2017; 51:12.

8. Drachler ML, Andresson MCS, Leite JCC, Marshall T, Aerts DRGC, Freitas PF, et al. Desigualdade social e outros determinantes da altura em crianças: uma análise multinível. Cad Saúde Pública 2003; 19:1815-25.

9. Neupane S, Doku DT. Neonatal mortality in Nepal: a multilevel analysis of a nationally representative. J Epidemiol Glob Health 2014; 4:213-22.

10. Ramos PCF, Carvalho Formiga MC, Costa CCL, Leon ACP. Diferenciais regionais da mortalidade da população infantil no estado do Rio Grande do Norte evidenciados pela utilização de modelos multiníveis. In: Anais Encontro Nacional de Estudos Populacionais. Belo Horizonte: Associação Brasileira de Estudos Populacionais; 2006. p 1-22.

11. Zanini RR, Moraes ABD, Giugliani ERJ, Riboldi J. Determinantes contextuais da mortalidade neonatal no Rio Grande do Sul por dois modelos de análise. Rev Saúde Pública 2011; 45:79-89.

12. Santos CAST, Amorim LDAF, Oliveira NF. Métodos de análise multinível em epidemiologia. In: Almeida Filho N, Barreto ML, organizadores. Epidemiologia \& saúde: fundamentos, métodos, aplicações. Rio de Janeiro: Guanabara Koogan; 2011. p. 265-72.

13. Puente-Palacios KE, Laros JA. Análise multinível: contribuições para estudos sobre efeito do contexto social no comportamento individual. Estud Psicol (Campinas) 2009; 26:349-61.

14. Diez-Roux AV, Aiello AE. Multilevel analysis of infectious diseases. J Infect Dis 2005; 191 Suppl 1:S25-33.

15. Tesfaye B, Atique S, Elias N, Dibaba L, Shabbir AS, Kebede M. Determinants and development of a web-based child mortality prediction model in resource-limited settings: a data mining approach. Comput Methods Programs Biomed 2017; 140:45-51.

16. Awiti JOA. A multilevel analysis of prenatal care and birth weight in Kenya. Health Econ Rev 2014; 4:33.

17. Maia LTS, Souza WV, Mendes ACG, Silva AGS. Uso do linkage para a melhoria da completude do SIM e do SINASC nas capitais brasileiras. Rev Saúde Pública 2017; 51:112.

18. Nascimento RM, Leite AJM, Almeida NMGS, Almeida PC, Silva CF. Determinantes da mortalidade neonatal: estudo caso-controle em Fortaleza, Ceará, Brasil. Cad Saúde Pública 2012; 28:559-72.

19. Silva ALA, Mendes ACG, Miranda GMD, Sá DA, Souza WV, Lyra TM. Avaliação da assistência hospitalar materna e neonatal: índice de completude da qualidade. Rev Saúde Pública 2014; 48:682-91.
20. Jain AK. Data clustering: 50 years beyond Kmeans. Pattern Recognit Lett 2010; 31:651-66.

21. Programa das Nações Unidas para o Desenvolvimento; Instituto de Pesquisa Econômica Aplicada; Fundação João Pinheiro. Atlas do desenvolvimento humano no Brasil. http://www. atlasbrasil.org.br/2013/pt/ (acessado em 20/ Mar/2018)

22. Organização Mundial da Saúde. Promoción del desarrollo fetal óptimo - informe de una reunión consultativa técnica. https://apps.who.int/iris/bitstream/handle/ 10665/43495/9243594001_spa.pdf (acessado em 23/Mar/2018).

23. Barros FC, Victora CG, Barros AJ, Santos IS, Albernaz E, Matijasevich A, et al. The challenge of reducing neonatal mortality in middle-income countries: findings from three Brazilian birth cohorts in 1982, 1993, and 2004. Lancet 2005; 365:847-54.

24. Gomes MRR, Costa JSD. Mortalidade infantil e as malformações congênitas no Município de Pelotas, RS, Brasil: estudo ecológico no período 1996-2008. Epidemiol Serv Saúde 2012; 21:119-28.

25. Maia LTS, Souza WV, Mendes ACG. Diferenciais nos fatores de risco para a mortalidade infantil em cinco cidades brasileiras: um estudo de caso-controle com base no SIM e no SINASC. Cad Saúde Pública 2012; 28:2163-76.

26. Mendes CQS, Avena MJ, Mandetta MA, Balieiro MMFG. Prevalência de nascidos vivos com anomalias congênitas no Município de São Paulo. Rev Soc Bras Enferm Pediatras (Online) 2015; 15:7-12.

27. Pereira APE, Leal MC, Gama SGN, Domingues RMSM, Schilithz AOC, Bastos MH. Determinação da idade gestacional com base em informações do estudo Nascer no Brasil. Cad Saúde Pública 2014; 30 Suppl:S59-70.

28. Polgliane RBS, Leal MDC, Amorim MHC, Zandonade E, Neto S. Adequação do processo de assistência pré-natal segundo critérios do Programa de Humanização do Pré-natal e Nascimento e da Organização Mundial de Saúde. Ciênc Saúde Colet 2014; 19:1999-2010.

29. Johnson RA, Wichern DW. Applied multivariate statistical analysis. 5 th Ed. New Jersey: Prentice Hall; 2014.

30. Instituto Brasileiro de Geografia e Estatística. Síntese de Indicadores Sociais: uma análise das condições de vida da população brasileira: 2012. Rio de Janeiro: Instituto Brasileiro de Geografia e Estatística; 2013.

31. Rayamajhi R, Thapa M, Pande S. The challenge of grandmultiparity in obstetric practice. Kathmandu University Medical Journal 2006; 4:70-4.

32. Silva ALA, Mendes ACG, Miranda GMD, Santos Neto PMD. Assistência ao parto no Brasil: uma situação crítica ainda não superada. 1999-2013. Rev Bras Saúde Mater Infant 2016; 16:129-37.

33. Lima LC. Idade materna e mortalidade infantil: efeitos nulos, biológicos ou socioeconômicos? Rev Bras Estud Popul 2013; 27:211-26. 
34. Villar J, Carroli G, Zavaleta N, Donner A, Wojdyla D, Faundes A, et al. Maternal and neonatal individual risks and benefits associated with caesarean delivery: multicentre prospective study. BMJ 2007; 335:1025.

35. Barros FC, Bhutta ZA, Batra M, Hansen TN, Victora CG, Rubens CE. Global report on preterm and stillbirth (3 of 7): evidence for effectiveness of interventions. BMC Pregnancy Childbirth 2010; 10 Suppl 1:S3.

36. Martins EF, Velásquez-Meléndez G. Determinantes da mortalidade neonatal a partir de uma coorte de nascidos vivos, Montes Claros, MG, 1997-1999. Rev Bras Saúde Mater Infant 2004; 4:405-12.

37. Zighelboim I, Sánchez W. Cesárea una panacea? Rev Obstet Ginecol Venezuela 2007; 67:217-21.

38. Oliveira RR, Melo EC, Novaes ES, Ferracioli PLRV, Mathias TAF. Fatores associados ao parto cesáreo nos sistemas público e privado de atenção à saúde. Rev Esc Enferm USP 2016; 50:734-41.

39. Silva ZP, Almeida MF, Ortiz LP, Alencar GP, Alencar AP, Schoeps D, et al. Morte neonatal precoce segundo complexidade hospitalar e rede SUS e não-SUS na Região Metropolitana de São Paulo, Brasil. Cad Saúde Pública 2010; 26:123-34

40. Leal MC, Gama SGN, Campos MR, Cavalini LT, Garbayo LS, Brasil CLP, et al. Fatores associados à morbi-mortalidade perinatal em uma amostra de maternidades públicas e privadas do Município do Rio de Janeiro, 1999-2001. Cad Saúde Pública 2004; 20 Suppl 1:S20-33.
41. Leal MC, Theme-Filha MM, Moura EC, Cecatti JG, Santos LMP. Atenção ao pré-natal e parto em mulheres usuárias do sistema público de saúde residentes na Amazônia Legal e no Nordeste, Brasil 2010. Rev Bras Saúde Matern Infant 2015; 15:91-104.

42. Guimarães MJB, Marques NM, Melo Filho DA, Szwarcwald CL. Condições de vida e mortalidade infantil: diferenciais intra-urbanos no Recife, Pernambuco, Brasil. Cad Saúde Pública 2003; 19:1413-24.

43. Carvalho AI, Buss PM. Determinantes sociais na saúde, na doença e na intervenção. In: Giovanella $\mathrm{L}$, organizador. Políticas e sistemas de saúde no Brasil. Rio de Janeiro: Editora Fiocruz; 2012. p. 121-42.

44. Bezerra Filho JG, Kerr LRFS, Miná DL, Barreto ML. Distribuição espacial da taxa de mortalidade infantil e principais determinantes no Ceará, Brasil, no período 2000-2002. Cad Saúde Pública 2007; 23:1173-85.

45. Ximenes RAA, Albuquerque MFPM, Souza WV, Montarroyos UR, Diniz GT, Luna CF, et al. Is it better to be rich in a poor area or poor in a rich area? A multilevel analysis of a casecontrol study of social determinants of tuberculosis. Int J Epidemiol 2009; 38:1285-96. 


\section{Abstract}

The study sought to identify individual and contextual risk factors in healthcare and their interactions and regional differences in the determination of infant mortality in Brazilian state capitals. This was a case-control study that analyzed 7,470 infant deaths in 2012 in the 27 state capitals, recorded in the Brazilian Mortality Information System (SIM) and matched with the Brazilian Information System on Live Births (SINASC) through linkage and 24,285 controls obtained by sampling the surviving liveborn infants from 2011 to 2012 from the total of 1,424,691 births. The individual explanatory variables corresponded to information available in the SINASC database, and the contextual variable consisted of a quality index for hospital care in the 702 healthcare services where the births occurred. A multilevel logistic model was used to analyze interaction. The principal determinants of infant mortality were biological factors (low birthweight, prematurity, congenital malformations, severe/moderate asphyxia, and race/color), mediated by maternal socioeconomic factors (schooling, marital status, and occupation) and insufficiency of prenatal care. Low number of prenatal visits was a risk factor for infant mortality, independently of the service's quality, except in the state capitals in the South of Brazil. In the interaction between income and prenatal care, few prenatal visits and birth in highincome state capitals showed a higher risk when compared to births in low-income state capitals $(\mathrm{OR}=0.68)$. Multilevel analysis evidenced regional inequalities in the risk models and reiterated the importance of biological determinants in the mediation of socioeconomic and healthcare factors in infant mortality.

Infant Mortality; Risk Factors; Social Determinants of Health; Multilevel Analysis

\section{Resumen}

El objetivo de la investigación fue identificar factores de riesgo individuales y contextuales de asistencia a la salud, sus interacciones y diferenciales regionales en la determinación de la mortalidad infantil en las capitales brasileñas. Se trata de un estudio caso-control, en el que se consideraron como casos los 7.470 óbitos infantiles ocurridos en 2012, en las 27 capitales del país, registrados en el Sistema de Informaciones sobre Mortalidad (SIM) y pareados con el Sistema de Información sobre Nacidos Vivos (SINASC) mediante linkage y 24.285 controles, obtenidos mediante muestra de los nacidos supervivientes entre 2011 y 2012, dentro de un universo de 1.424.691 nacimientos. Las variables explicativas a nivel individual corespondieron a la información proporcionada por el SINASC, y la variable contextual consistió en un indice de calidad de la asistencia hospitalaria, referente a los 702 servicios de salud donde se produjeron los nacimientos. Se empleó el modelo logístico multinivel y un análisis de interacción. Los principales determinantes de la mortalidad infantil fueron los factores biológicos (bajo peso al nacer, prematuridad, malformación congénita, asfixia grave/moderada y raza/color), mediados por los socioeconómicos maternos (escolaridad, estado civil y ocupación), y por la insuficiencia del prenatal. Realizar un bajo número de consultas prenatales representó un riesgo para la mortalidad infanti, independientemente de la calidad del servicio, a excepción de las capitales de la Región Sur. En la interacción entre renta y la atención prenatal, se observó que realizar pocas consultas y nacer en ciudades con alta renta representó un riesgo mayor cuando lo comparamos con los nacimientos en capitales con renta baja $(O R=0,68)$. El análisis multinivel evidenció desigualdades regionales en los modelos de riesgo y reiteró la importancia de los determinantes biológicos con la mediación de factores socioeconómicos y asistenciales en la mortalidad infantil.

Mortalidad Infantil; Factores de Riesgo; Determinantes Sociales de la Salud; Análisis Multinivel
Recebido em 24/Mar/2019

Versão final reapresentada em 21/Jun/2019

Aprovado em 14/Ago/2019 